\title{
Formation of Alkynylgermyl-Substituted Germylenes via a Catenation of Ge Atoms
}

Chenghuan Liu, ${ }^{\mathrm{a}}$ Keke Zhu, ${ }^{\mathrm{a}}$ Weichun Han, ${ }^{\mathrm{a}}$ Xue Liu, ${ }^{\mathrm{a}}$ Zheng-Feng Zhang, ${ }^{\mathrm{c}}$ Ming-Der Su, ${ }^{* \mathrm{c}, \mathrm{d}} \mathrm{Di}$ $\mathrm{Wu}^{* \mathrm{~b}}$ and Yan $\mathrm{Li}^{* \mathrm{a}}$

a Key Laboratory of Organosilicon Chemistry and Material Technology of Ministry of Education, Hangzhou Normal University, Hangzhou, 311121, China

${ }^{b}$ College of Material, Chemistry and Chemical Engineering, Hangzhou Normal University, Hangzhou, 311121, China.

${ }^{c}$ Department of Appied Chemistry, National Chiayi University, Chiayi, 60004, Taiwan

${ }^{d}$ Department of Medicinal and Applied Chemistry, Kaohsiung Medical University, Kaohsiung, 80708, Taiwan

\section{Content:}

\section{X-Ray crystallographic details}

II. Theoretical calculations

III. Collected ${ }^{1} \mathrm{H}$ and/or ${ }^{13} \mathrm{C},{ }^{29} \mathrm{Si}$ NMR spectra of compounds $1,2,3,4$ IV. References 


\section{X-Ray crystallographic details}

Crystallographic data for compounds $\mathbf{1}_{0.5}$.hexane, 2, 3 and 4 were collected on an Smart Apex II DUO system. During measurements a graphite-monochromatic Mo-K $K_{\alpha}$ radiation $(\lambda=0.71073 \AA)$ was applied for $\mathbf{1}_{0.5}$.hexane, 2, 3 and 4 . Absorption corrections were all employed using the spherical harmonics program (multi-scan type). All the structures were solved by direct methods $(\text { SHELXT) })^{\left[S_{1}\right]}$ and refined against $F^{2}$ using SHELXL. ${ }^{\left[\mathrm{S}_{2}\right]}$ In general, the non-hydrogen atoms were located by difference Fourier synthesis and refined anisotropically, and hydrogen atoms were included using a riding mode with $U_{\text {iso }}$ tied to the $U_{\text {iso }}$ of the parent atoms unless otherwise specified. In 3, one TMS group was located in disorder and treated by the PART method, where final refinement gave two parts as $\mathrm{C}(029) \mathrm{C}(02 \mathrm{~A}) \mathrm{C}(4)$ with occupation of $0.713(8)$ and $\mathrm{C}(4 \mathrm{~A}) \mathrm{C}(\mathrm{o}) \mathrm{C}(\mathrm{o} 2 \mathrm{~B})$ with occupation of $0.287(8)$. A summary of cell parameters, data collection, structure solution and refinements is given in Table S1.

Table S1. Crystal data and refinements

\begin{tabular}{lllll}
\hline & $\mathbf{1} \cdot 0.5 n$-hexane & $\mathbf{2}$ & $\mathbf{3}$ & \multicolumn{1}{c}{} \\
\hline Empirical formula & $\mathrm{C}_{33} \mathrm{H}_{57} \mathrm{Ge}_{2} \mathrm{~N}_{2} \mathrm{Si}_{3}$ & $\mathrm{C}_{58} \mathrm{H}_{90} \mathrm{Ge}_{3} \mathrm{~N}_{4} \mathrm{Si}_{4}$ & $\mathrm{C}_{74} \mathrm{H}_{108} \mathrm{Ge}_{2} \mathrm{~N}_{4} \mathrm{Si}_{4}$ & $\mathrm{C}_{33} \mathrm{H}_{59} \mathrm{Ge}_{2} \mathrm{~N}_{3} \mathrm{Si}_{4}$ \\
formula weight & 711.25 & 1173.47 & 1311.18 & 955.37 \\
crystal system & Monoclinic & Monoclinic & Triclinic & Triclinic \\
space group & $P 2_{1} / \mathrm{n}$ & $C 2 / \mathrm{c}$ & $P-1$ & $P-1$ \\
$a / \AA$ & $12.140(4)$ & $46.758(3)$ & $12.5928(13)$ & $12.035(6)$ \\
$b / \AA$ & $16.494(5)$ & $12.5284(9)$ & $14.6796(16)$ & $13.238(7)$ \\
$\mathrm{c} / \AA$ & $21.272(7)$ & $22.5579(16)$ & $23.294(3)$ & $15.489(8)$ \\
$\alpha /$ deg & 90 & 90.00 & $96.898(2)$ & $90.886(9)$ \\
$\beta /$ deg & $99.538(7)$ & $102.0450(10)$ & $95.866(2)$ & $106.918(9)$ \\
$\gamma /$ deg & 90 & 90.00 & $113.270(2)$ & $103.615(10)$ \\
$V / \AA^{3}$ & $1590.87(14)$ & $12923.6(16)$ & $3873.9(7)$ & $2285(2)$ \\
$Z$ & 4 & 8 & 2 & 2 \\
$\rho_{\text {calcd }} / \mathrm{g} \cdot \mathrm{cm}-3$ & 1.125 & 1.206 & 1.124 & 1.098 \\
$\mu /$ mm & -1 & 1.497 & 0.877 & 1.442 \\
$F(000)$ & 1.537 & 4944 & 1400.0 & 796 \\
$\theta$ range/deg & 1500.0 & $1.69-28.37$ & $1.786-56.862$ & $1.59-27.00$ \\
index ranges & $3.05-56.84$ & $-60 \leq h \leq 62$ & $-16 \leq h \leq 16$ & $-14 \leq h \leq 15$ \\
& $-14 \leq h \leq 16$ & $-16 \leq k \leq 16$ & $-19 \leq k \leq 17$ & $-16 \leq k \leq 16$ \\
& $-14 \leq k \leq 21$ & $-30 \leq l \leq 28$ & $-31 \leq l \leq 31$ & $-17 \leq l \leq 19$ \\
collected data & $-28 \leq l \leq 27$ & 53194 & 36065 & 18801 \\
unique data & 34782 & 16084 & 18251 & 9542 \\
data/restraints/paramete & 10464 & $\left(R_{\text {int }}=0.0762\right)$ & $\left(R_{\text {int }}=0.0283\right)$ & $\left(R_{\text {int }}=0.0327\right)$ \\
rs & $\left(R_{\text {int }}=0.1299\right)$ & $16084 / 0 / 634$ & $18251 / 3 / 816$ & $5942 / 0 / 397$ \\
GOF on $F^{2}$ & 0.970 & 0.986 & 1.053 & 0.981 \\
& & & &
\end{tabular}




\begin{tabular}{|c|c|c|c|c|}
\hline $\begin{array}{l}\text { final } R \text { indices }[I> \\
2(I)]\end{array}$ & $\begin{array}{l}R_{1}=0.0540 \\
w R_{2}=0.1047\end{array}$ & $\begin{array}{l}R_{1}=0.0474 \\
w R_{2}=0.0916\end{array}$ & $\begin{array}{l}R_{1}=0.0505 \\
w R_{2}=0.1161\end{array}$ & $\begin{array}{l}R_{1}=0.0441 \\
w R_{2}=0.1130\end{array}$ \\
\hline$R$ indices (all data) & $\begin{array}{l}R_{1}=0.1659 \\
w R_{2}=0.1375\end{array}$ & $\begin{array}{l}R_{1}=0.0942 \\
w R_{2}=0.1064\end{array}$ & $\begin{array}{l}R_{1}=0.0717 \\
w R_{2}=0.1261\end{array}$ & $\begin{array}{l}R_{1}=0.0742 \\
w R_{2}=0.1231\end{array}$ \\
\hline $\begin{array}{l}\text { Largest diff peak/hole } \\
\left(\mathrm{e} \cdot \AA^{-3}\right)\end{array}$ & $0.34 /-0.34$ & $0.531 /-0.494$ & $2.52 /-1.63$ & $0.450 /-0.490$ \\
\hline
\end{tabular}

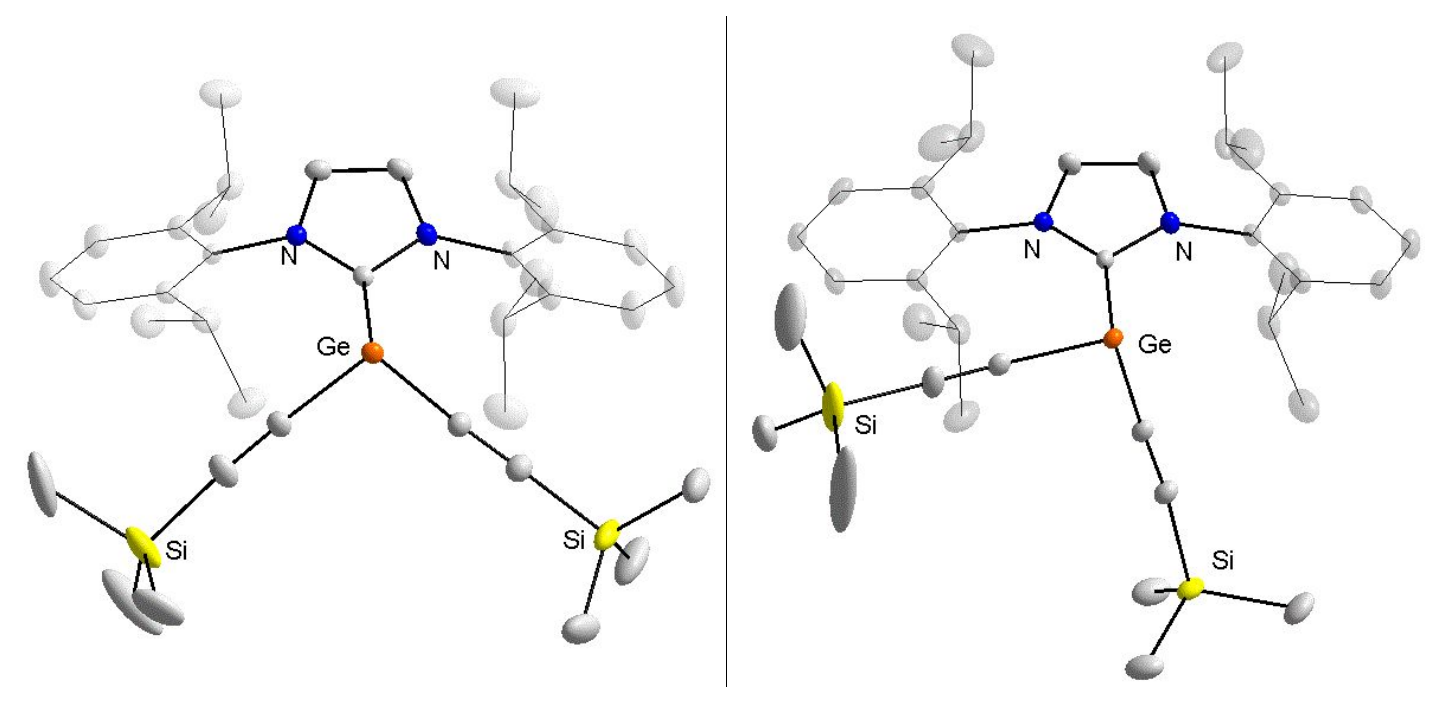

Figure S1. Two types of molecular configuration of compound $\mathbf{3}$ in one asymmetric unit $(\mathrm{H}$ atoms are omitted for clarity). 
II. Collected ${ }^{1} \mathrm{H},{ }^{13} \mathrm{C}$ and ${ }^{29} \mathrm{Si}$ NMR spectra of compounds 1-4

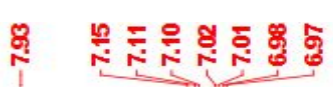

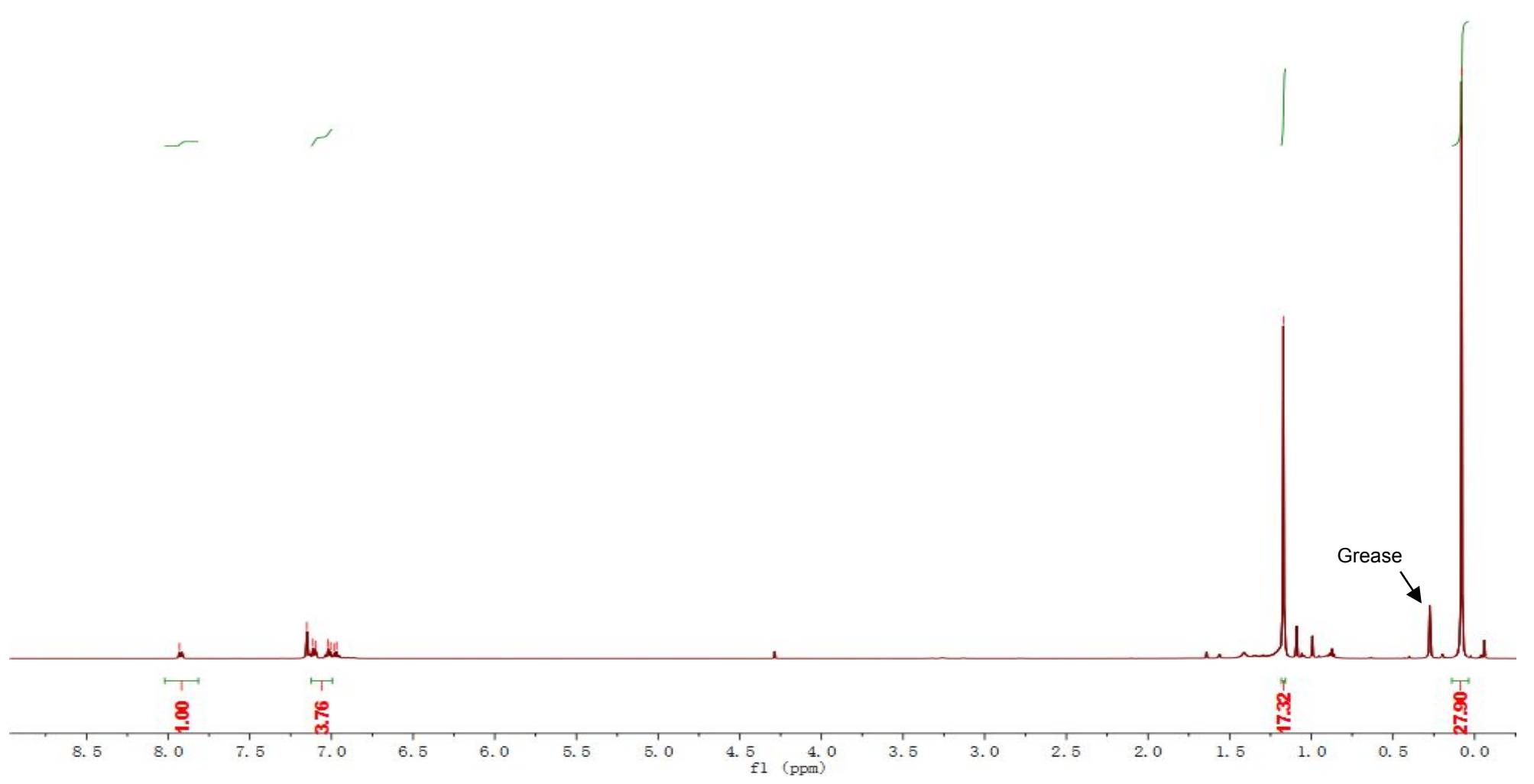

Figure S2. ${ }^{1} \mathrm{H}$ NMR spectrum of 1 in $\mathrm{C}_{6} \mathrm{D}_{6}$ 


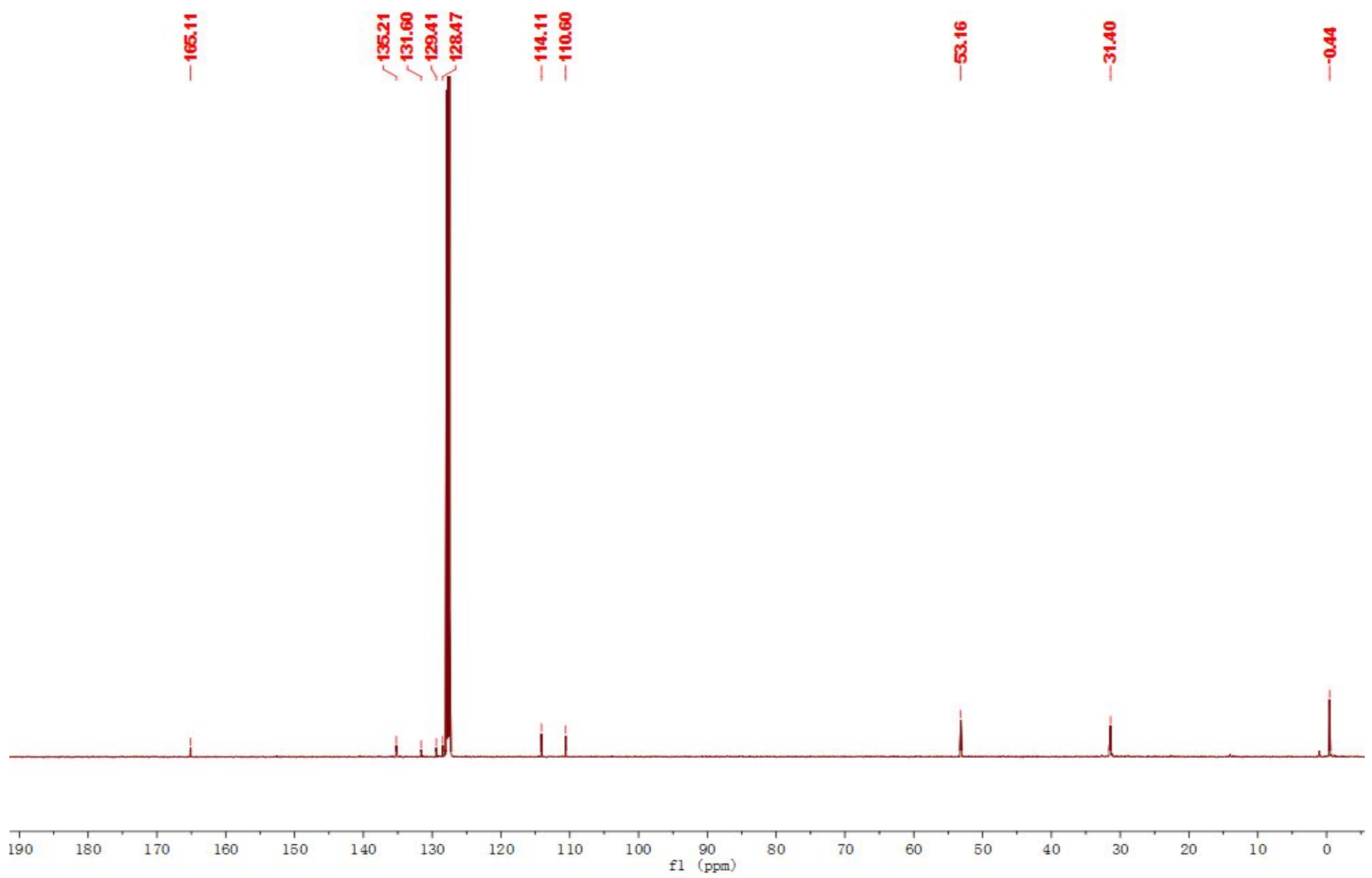

Figure S3. ${ }^{13} \mathrm{C}\left\{{ }^{1} \mathrm{H}\right\}$ NMR spectrum of $\mathbf{1}$ in $\mathrm{C}_{6} \mathrm{D}_{6}$ 


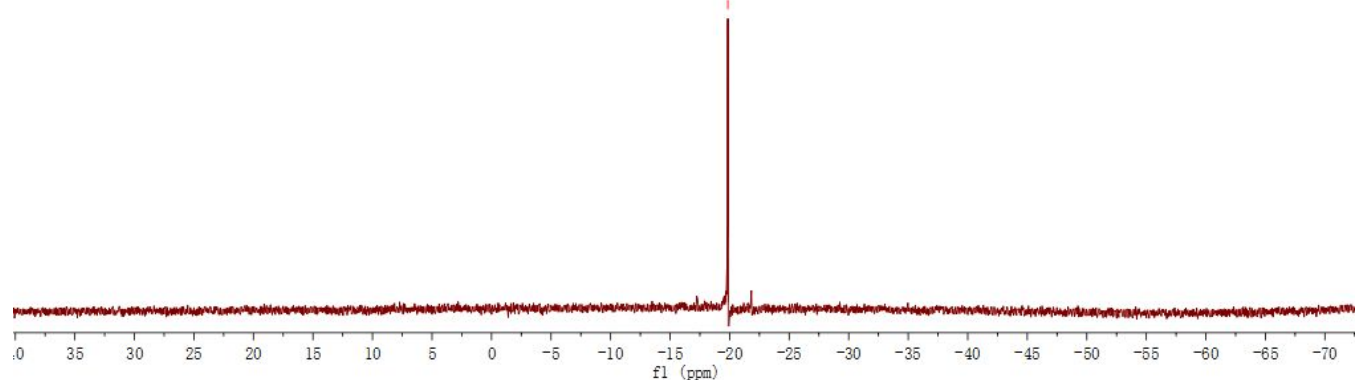

Figure S4. ${ }^{29} \mathrm{Si}$ NMR spectrum of $\mathbf{1}$ in $\mathrm{C}_{6} \mathrm{D}_{6}$ 


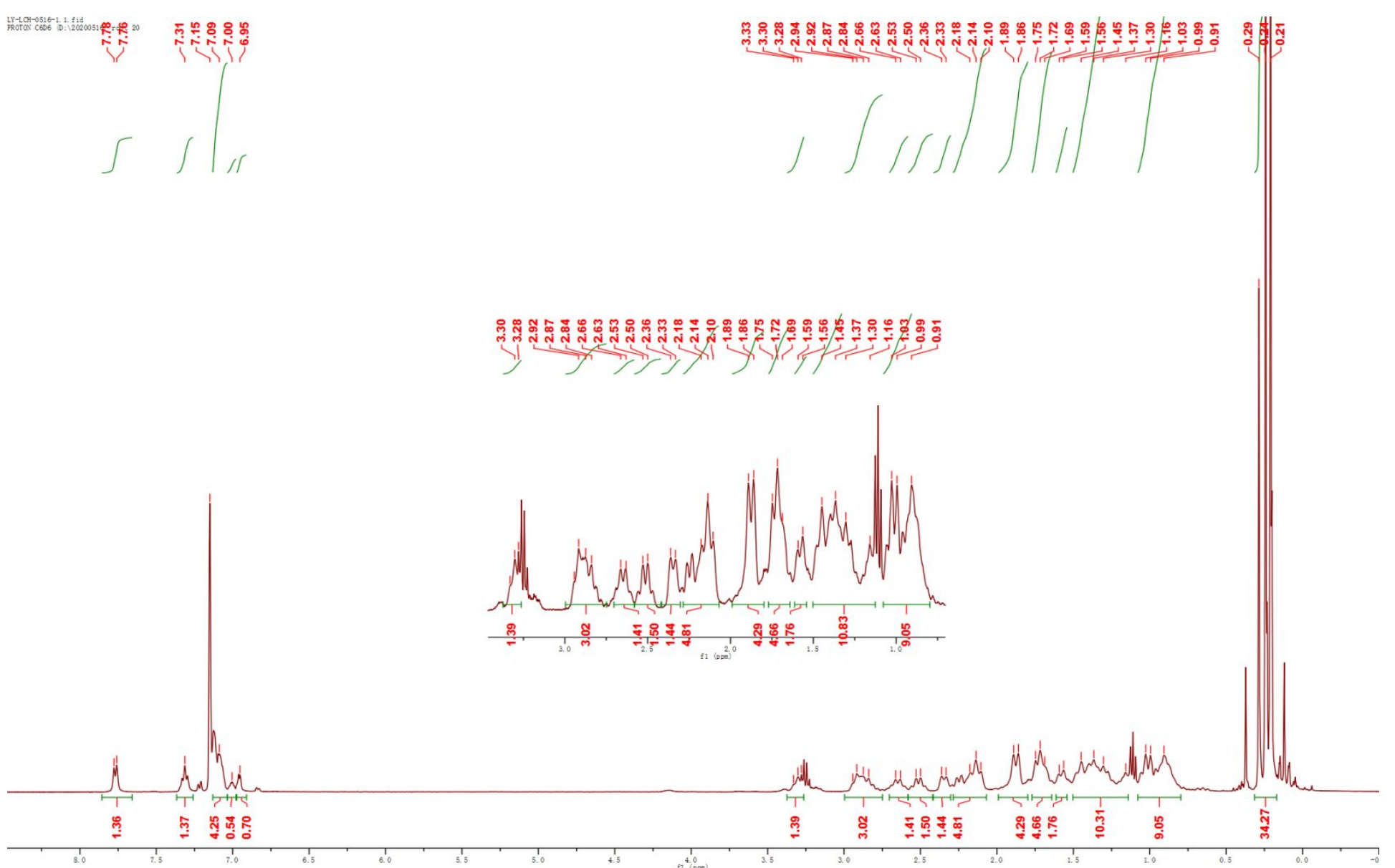

Figure S5. ${ }^{1} \mathrm{H}$ NMR spectrum of 2 in $\mathrm{C}_{6} \mathrm{D}_{6}$ 


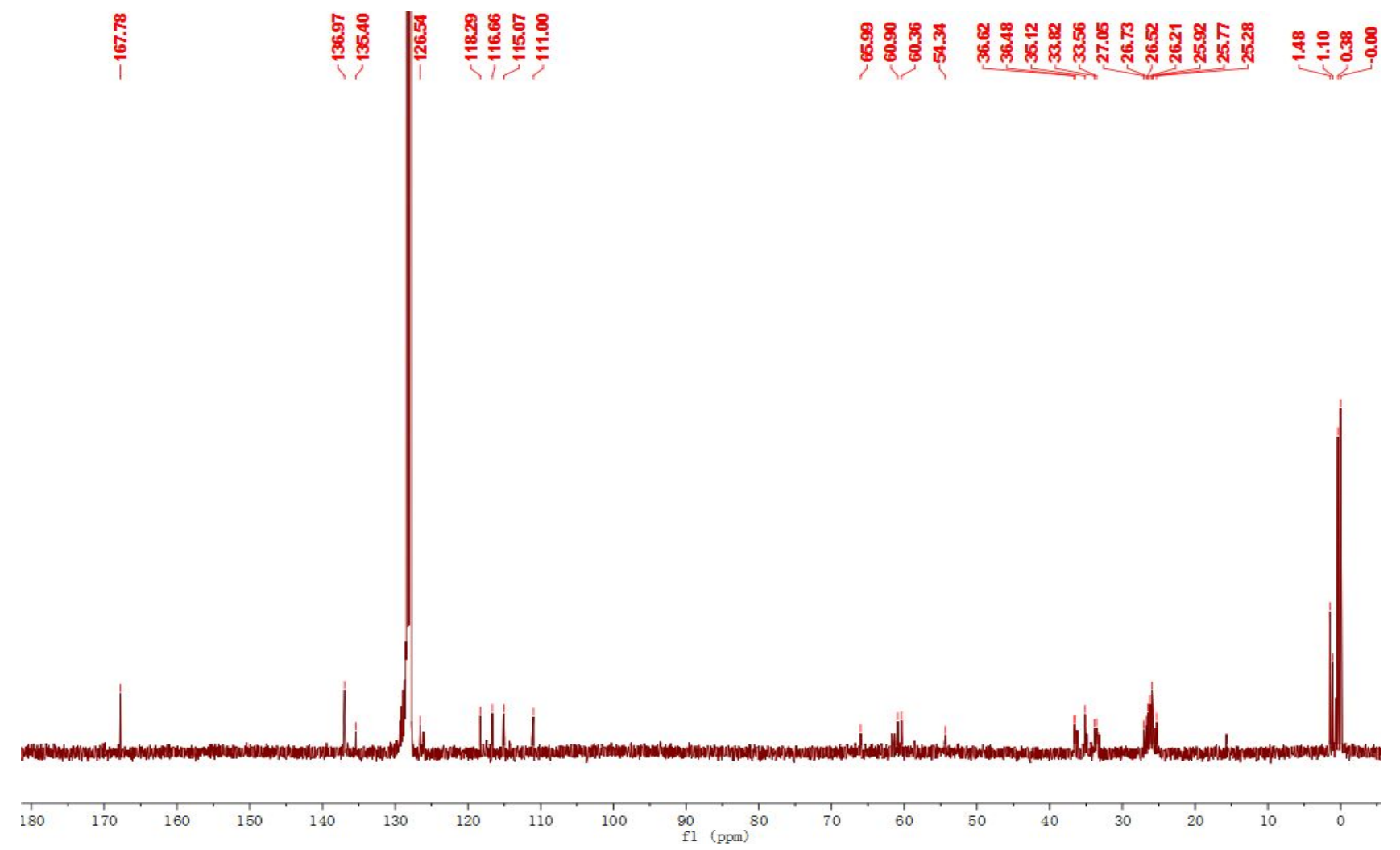

Figure S6. ${ }^{13} \mathrm{C}\left\{{ }^{1} \mathrm{H}\right\}$ NMR spectrum of $\mathbf{2}$ in $\mathrm{C}_{6} \mathrm{D}_{6}$ 


\section{ร}

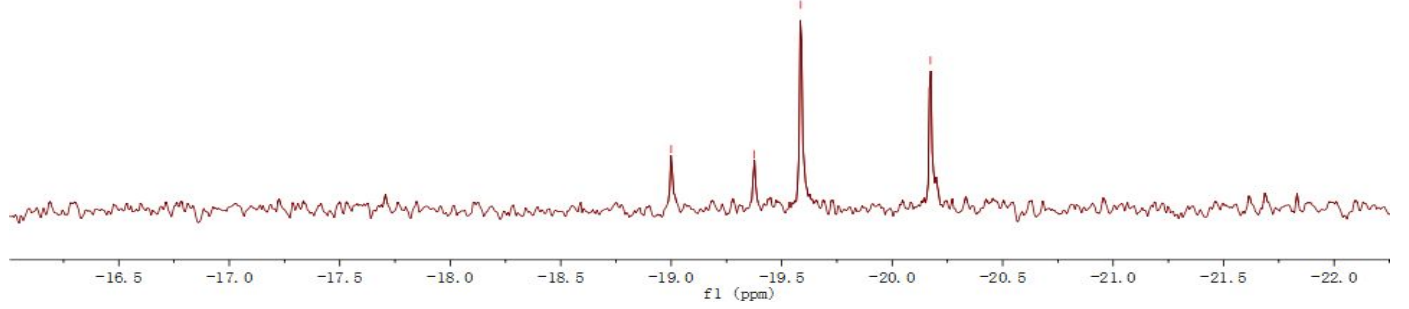

Figure S7. ${ }^{29} \mathrm{Si}$ NMR spectrum of 2 in $\mathrm{C}_{6} \mathrm{D}_{6}$ 


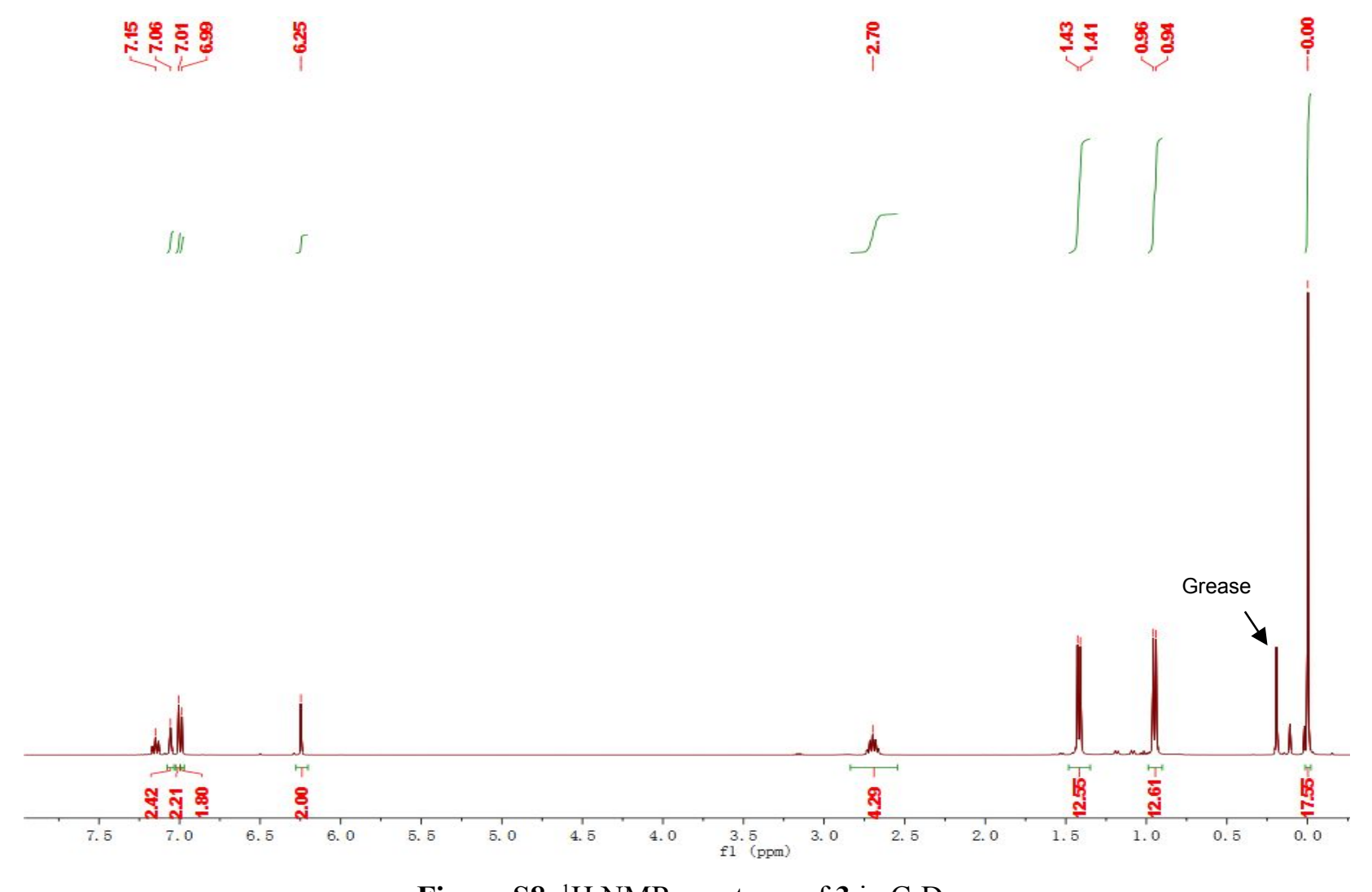

Figure S8. ${ }^{1} \mathrm{H}$ NMR spectrum of 3 in $\mathrm{C}_{6} \mathrm{D}_{6}$ 


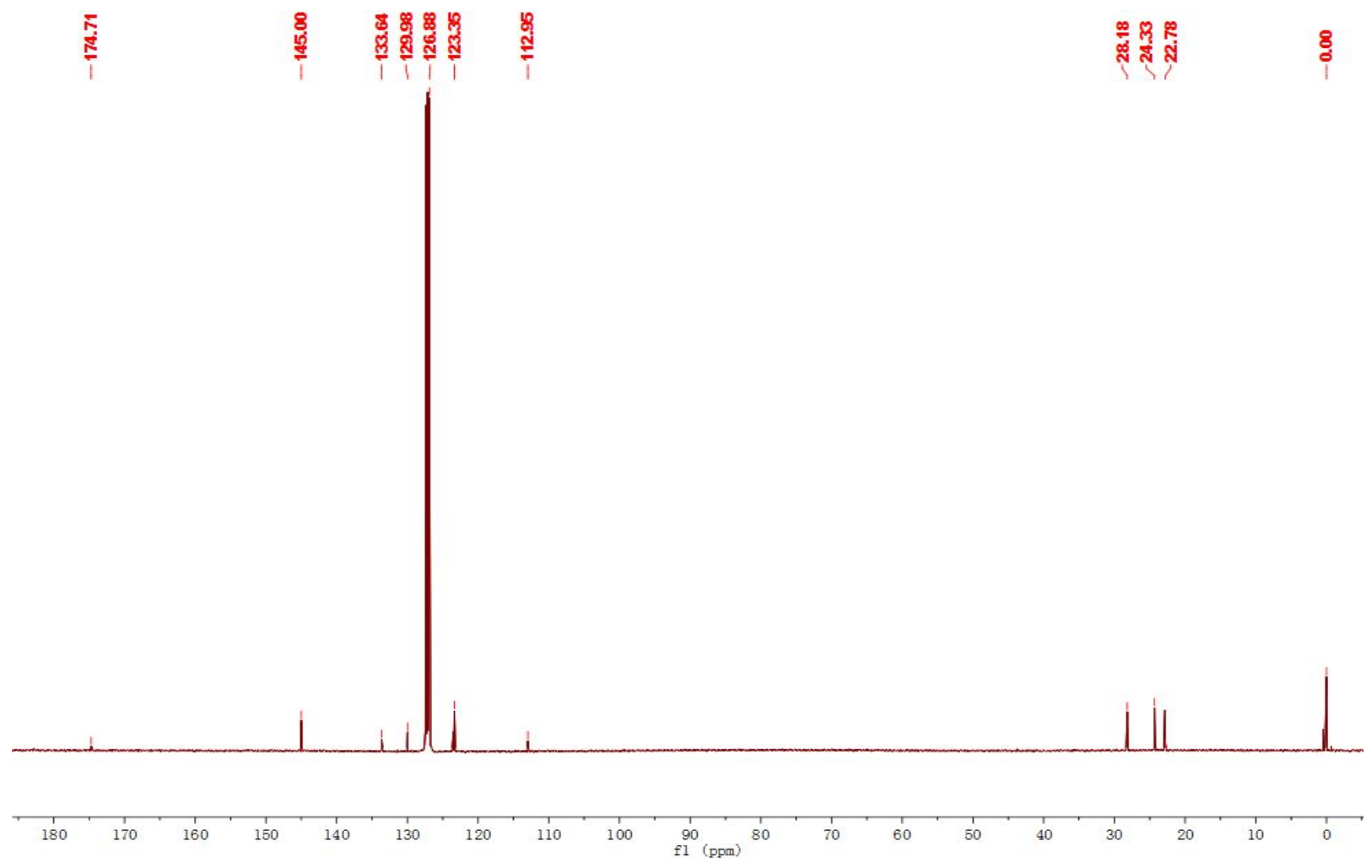

Figure S9. ${ }^{13} \mathrm{C}\left\{{ }^{1} \mathrm{H}\right\}$ NMR spectrum of 3 in $\mathrm{C}_{6} \mathrm{D}_{6}$ 

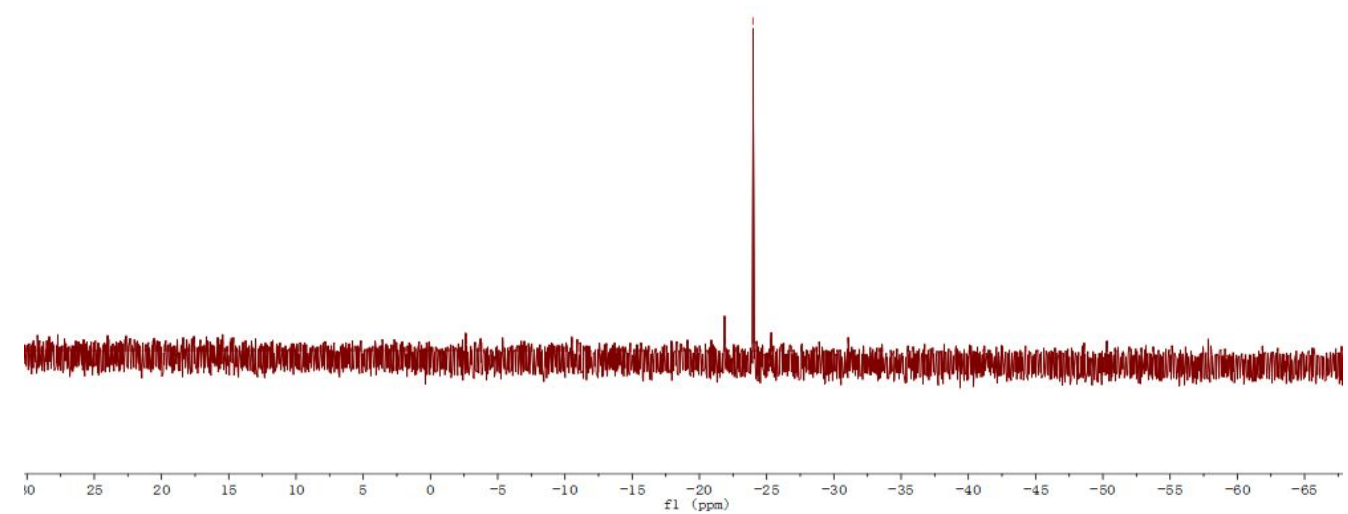

Figure S10. ${ }^{29} \mathrm{Si}$ NMR spectrum of $\mathbf{3}$ in $\mathrm{C}_{6} \mathrm{D}_{6}$ 


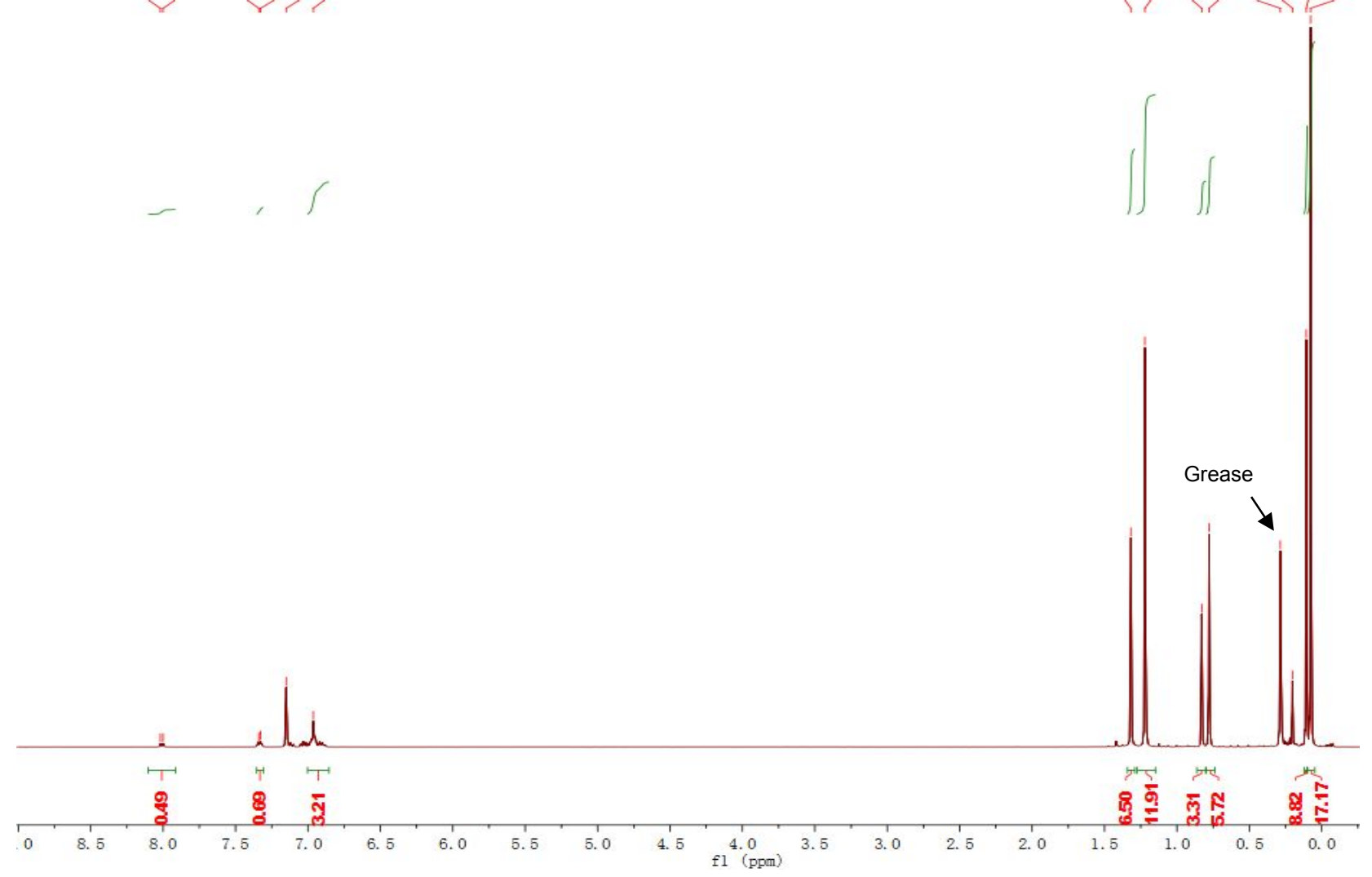

Figure S11. ${ }^{1} \mathrm{H}$ NMR spectrum of 4 in $\mathrm{C}_{6} \mathrm{D}_{6}$ 


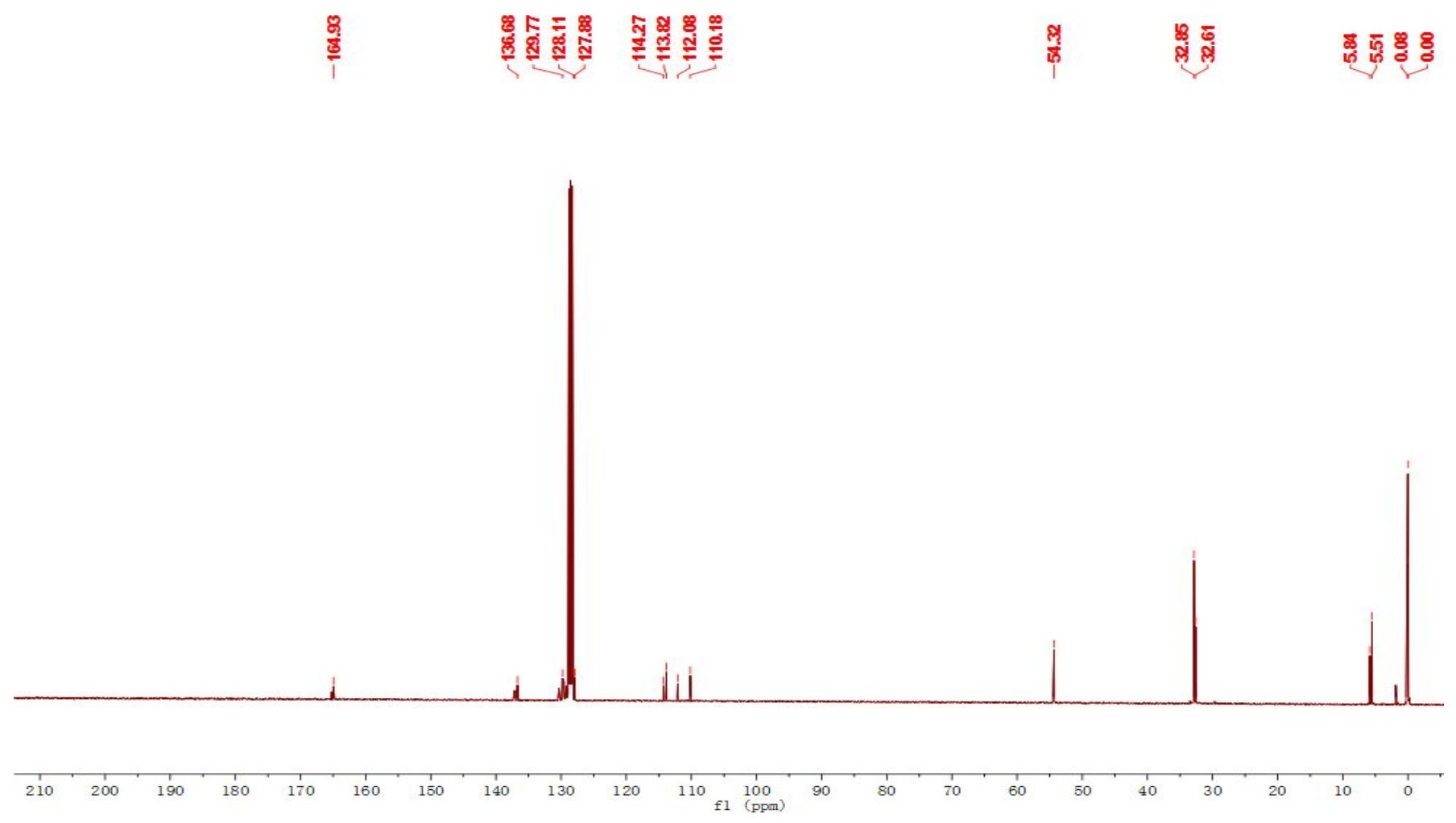

Figure S12. ${ }^{13} \mathrm{C}\left\{{ }^{1} \mathrm{H}\right\}$ NMR spectrum of 4 in $\mathrm{C}_{6} \mathrm{D}_{6}$ 


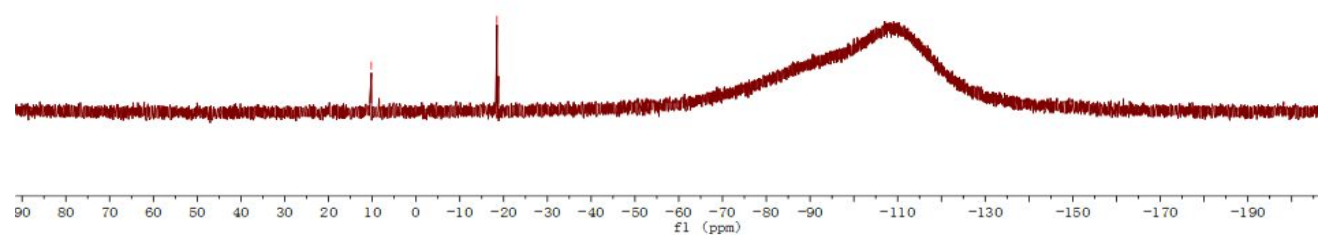

Figure S13. ${ }^{29} \mathrm{Si}$ NMR spectrum of 4 in $\mathrm{C}_{6} \mathrm{D}_{6}$ 


\section{Theoretical calculations}

Methods: In this work, geometry optimizations were carried out without any symmetry restriction by means of density functional theory with the M06-2X functional. This functional used in this work is due to the fact that it has good performance for the fields concerning thermochemistry, kinetics, and non-covalent interactions (such as van der Waals interactions) in main group elements. ${ }^{[\mathrm{S} 3]}$ The basis sets with split valence polarization (def2-SVP) ${ }^{[\mathrm{S} 4]}$ from Ahlrichs and coworkers was applied for all atoms. The Berny analytical gradient optimization method using GEDIIS ${ }^{[S 5]}$ as implemented in the Gaussian09 program ${ }^{[\mathrm{S6}]}$ was employed in geometry optimization steps. Harmonic vibrational frequency calculations were performed on all structures to verify the nature of the stationary points located on the potential energy surface. That is, the local minimum (no imaginary frequencies) and the transition state (one imaginary frequency). The normal modes corresponding to the imaginary frequencies in the transition state structures are related to the vibrations of new forming bonds. In the free energy profile, we report $\Delta \mathrm{G}$ (the Gibbs free energies) at $298.15 \mathrm{~K}$ and $1 \mathrm{~atm}$. The minimal and transition states were located by DFT at M06-2X/def2-SVP level. The single point with SMD model ${ }^{[\mathrm{S4}]}$ was performed to take solvation effects (Et $\mathrm{E}_{2} \mathrm{O}$ ) into account at M06-2X/def2-TZVP[S7]//M06-2X/def2-SVP level.

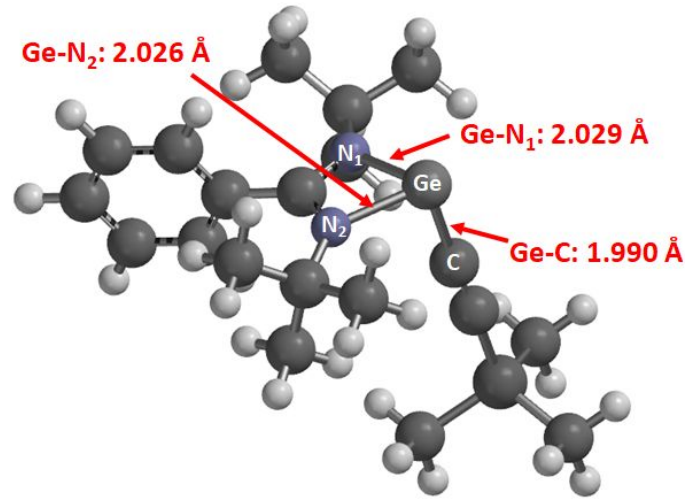

$\mathrm{L}_{1} \mathrm{GeC} \equiv \mathrm{CTMS}$

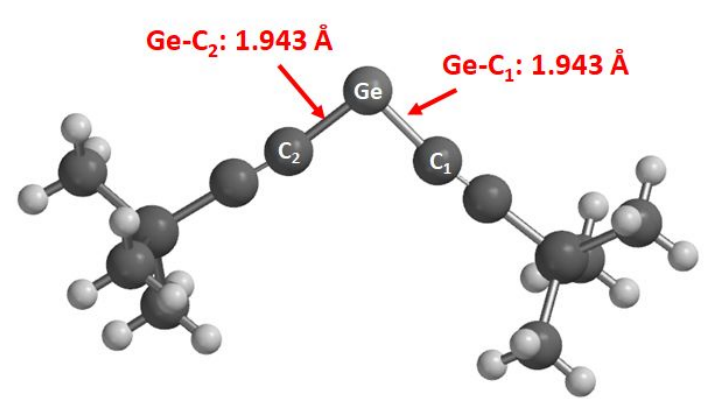

$\operatorname{Ge}(\mathrm{C} \equiv \mathrm{CTMS})_{2}$ 


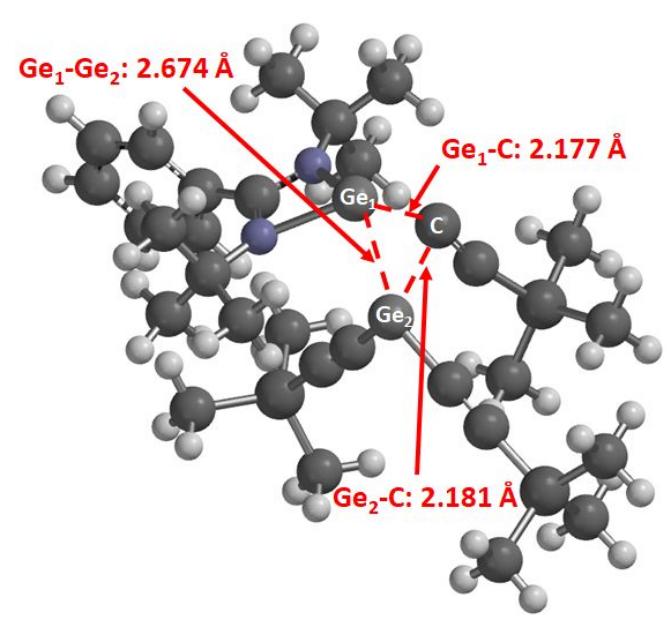

TS1

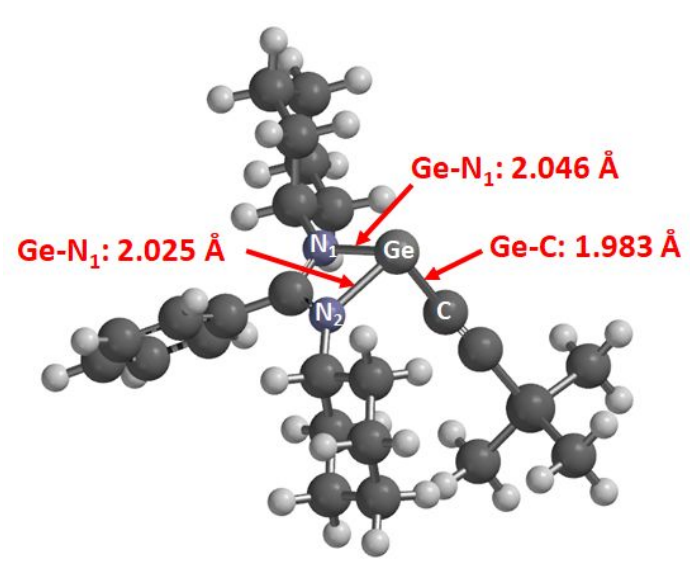

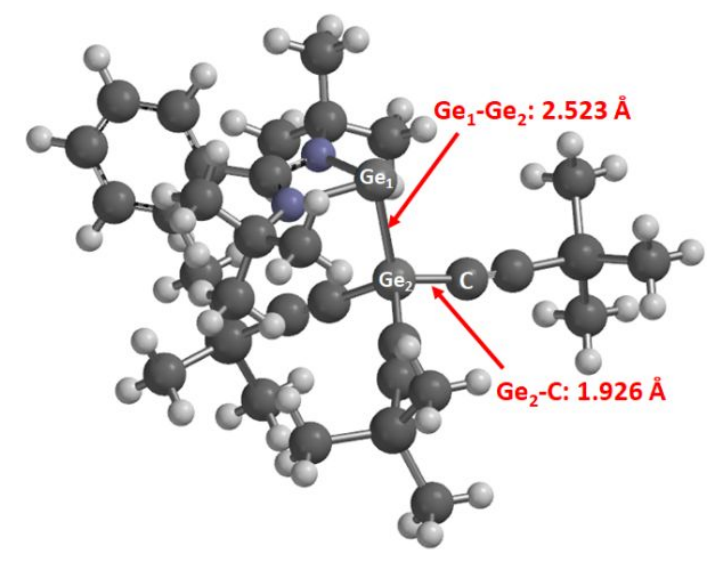

1

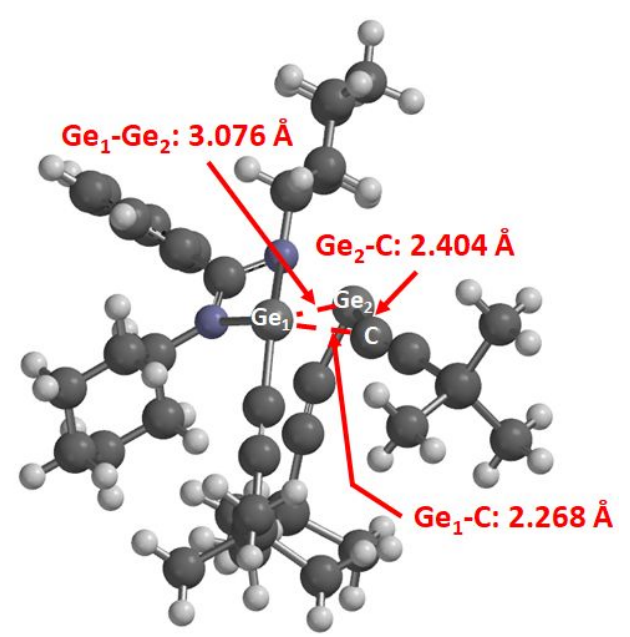




\section{$\mathrm{L}_{2} \mathrm{GeC} \equiv \mathrm{CTMS}$}

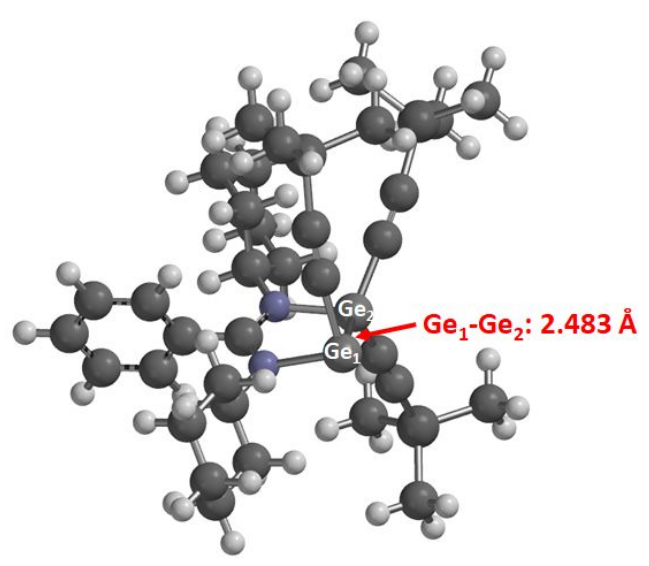

Int
TS2

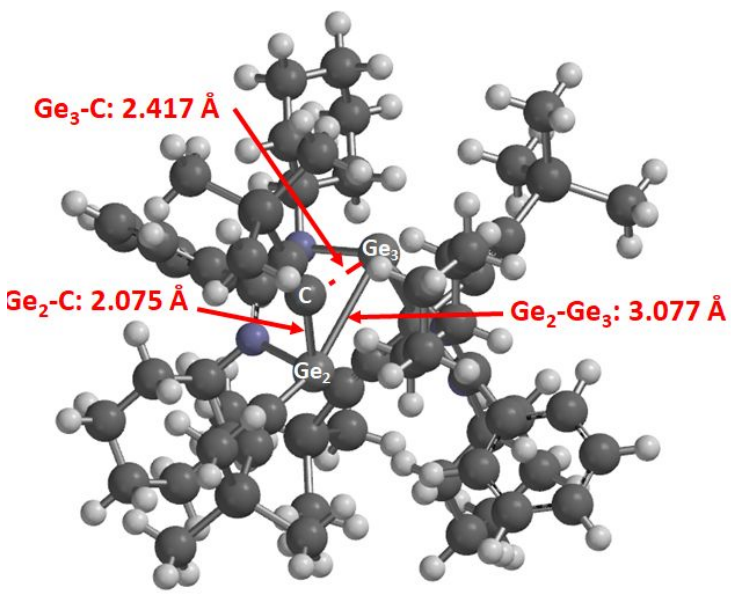

TS3 


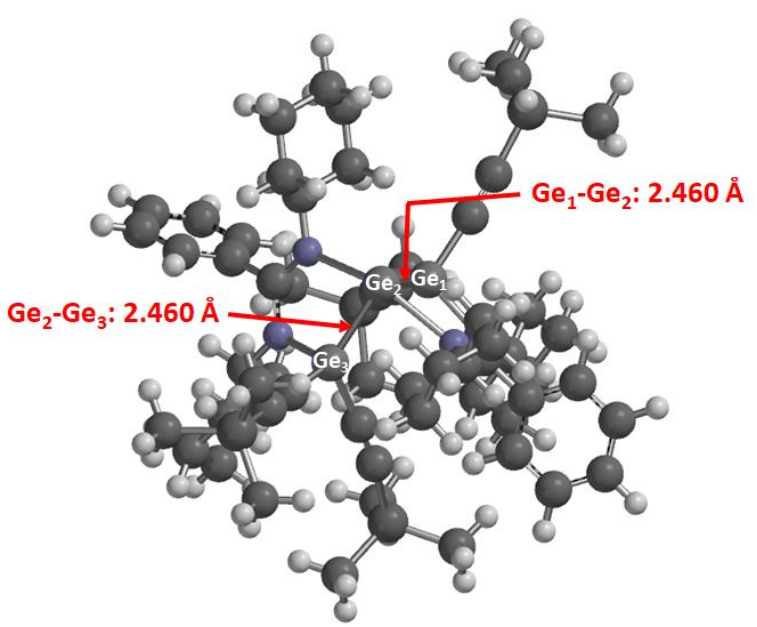

2

Figure S14. Selected optimized geometries of the minimal and transition states for formation of compounds 1 and 2 at the M06-2X/def2-SVP level of theory.

Table S2

M06-2X/def2-SVP

Ge-(CCTMS $)_{2}$

ENERGY (WITHOUT THE ZERO-POINT) $=-3046.89275781$ Hartree

FREE ENERGY = -3046.700925 Hartree

\begin{tabular}{llrr} 
Atomic & \multicolumn{3}{c}{ Coordinates (Angstroms) } \\
Number & $\mathrm{X}$ & $\mathrm{Y}$ & $\mathrm{Z}$
\end{tabular}




$\begin{array}{rrrr}32 & 0.000005 & 2.240215 & 0.000007 \\ 14 & 3.982880 & -0.813944 & 0.001243 \\ 14 & -3.982905 & -0.813893 & -0.001237 \\ 6 & -1.446461 & 0.942668 & -0.005597 \\ 6 & -2.462236 & 0.253619 & -0.008898 \\ 6 & -3.592553 & -2.409264 & -0.909350 \\ 1 & -3.302049 & -2.204964 & -1.950024 \\ 1 & -4.471919 & -3.070949 & -0.920672 \\ 1 & -2.763913 & -2.942289 & -0.421458 \\ 6 & -5.365359 & 0.119027 & -0.863550 \\ 1 & -5.572767 & 1.070304 & -0.352725 \\ 1 & -6.289195 & -0.479321 & -0.867492 \\ 1 & -5.097059 & 0.342675 & -1.906227 \\ 6 & -4.426678 & -1.153885 & 1.790984 \\ 1 & -3.606148 & -1.675080 & 2.304814 \\ 1 & -5.326371 & -1.785300 & 1.848336 \\ 1 & -4.628346 & -0.216797 & 2.329396 \\ 6 & 1.446495 & 0.942694 & 0.005568 \\ 6 & 2.462231 & 0.253591 & 0.008942 \\ 6 & 4.427609 & -1.152705 & -1.790974 \\ 1 & 3.607277 & -1.673176 & -2.305835 \\ 1 & 5.327128 & -1.784383 & -1.848199 \\ 1 & 4.630000 & -0.215241 & -2.328466 \\ 6 & 3.592064 & -2.409919 & 0.908117 \\ 1 & 3.300745 & -2.206235 & 1.948682 \\ & & & \end{array}$




$\begin{array}{llrl}1 & 4.471460 & -3.071556 & 0.919685 \\ 1 & 2.763834 & -2.942671 & 0.419237 \\ 6 & 5.364908 & 0.118498 & 0.864744 \\ 1 & 5.572470 & 1.070084 & 0.354550 \\ 1 & 6.288782 & -0.479783 & 0.868765 \\ 1 & 5.096149 & 0.341562 & 1.907423\end{array}$

\section{Table S3}

\section{M06-2X/def2-SVP}

$\mathrm{L}_{1} \mathrm{Ge}-\mathrm{CCTMS}\left(\mathrm{L}_{1}=\mathrm{PhC}(\mathrm{N} t \mathrm{Bu})_{2}\right)$

ENERGY (WITHOUT THE ZERO-POINT) $=$-3255.98680739 Hartree

FREE ENERGY = -3255.573244 Hartree

\begin{tabular}{rrrr} 
Atomic & \multicolumn{3}{c}{ Coordinates (Angstroms) } \\
Number & $\mathrm{X}$ & $\mathrm{Y}$ & $\mathrm{Z}$ \\
\hline 32 & 0.329142 & -0.079949 & 2.176977 \\
14 & 4.220982 & 0.044025 & -1.057617 \\
7 & -0.841597 & 1.042844 & 0.958232 \\
7 & -0.844888 & -1.122579 & 0.896065 \\
6 & -1.370705 & -0.022948 & 0.374228 \\
6 & -0.956862 & 2.473107 & 0.677959 \\
6 & -0.188315 & 2.811665 & -0.607074 \\
1 & -0.672810 & 2.351571 & -1.479864
\end{tabular}




$\begin{array}{lrrr}1 & 0.842421 & 2.433848 & -0.536626 \\ 1 & -0.164722 & 3.900483 & -0.764908 \\ 6 & -0.289072 & 3.181274 & 1.860835 \\ 1 & -0.813761 & 2.947226 & 2.799028 \\ 1 & -0.298548 & 4.270154 & 1.712956 \\ 1 & 0.760161 & 2.858300 & 1.956378 \\ 6 & -2.412303 & 2.943926 & 0.567442 \\ 1 & -2.897552 & 2.570008 & -0.344038 \\ 1 & -2.439462 & 4.043226 & 0.535561 \\ 1 & -2.993270 & 2.608555 & 1.439661 \\ 6 & -2.422955 & 0.014800 & -0.686279 \\ 6 & -3.773611 & -0.028776 & -0.326073 \\ 1 & -4.046097 & -0.103494 & 0.728640 \\ 6 & -4.759163 & 0.025675 & -1.308092 \\ 1 & -5.811841 & -0.005710 & -1.023389 \\ 6 & -4.400962 & 0.122110 & -2.653649 \\ 1 & -5.174032 & 0.167013 & -3.422177 \\ 6 & -3.055374 & 0.160779 & -3.015376 \\ 1 & -2.773039 & 0.232835 & -4.066593 \\ 6 & -2.065918 & 0.108022 & -2.033648 \\ 1 & -1.009345 & 0.136557 & -2.309110 \\ 6 & -0.943883 & -2.529921 & 0.511712 \\ 6 & -0.293724 & -2.747467 & -0.861746 \\ 1 & -0.874804 & -2.253659 & -1.653410 \\ 1 & -0.247311 & -3.821650 & -1.096134 \\ 1 & 0.726266 & -2.335077 & -0.860421\end{array}$




$\begin{array}{lrrr}6 & -0.147861 & -3.303158 & 1.567541 \\ 1 & 0.896983 & -2.953678 & 1.588518 \\ 1 & -0.144937 & -4.377702 & 1.336952 \\ 1 & -0.587286 & -3.159667 & 2.566062 \\ 6 & -2.391526 & -3.035476 & 0.503877 \\ 1 & -2.883850 & -2.804028 & 1.460189 \\ 1 & -2.401245 & -4.126680 & 0.364051 \\ 1 & -2.975068 & -2.587393 & -0.311422 \\ 6 & 1.842829 & -0.042628 & 0.886090 \\ 6 & 2.812515 & -0.014840 & 0.134684 \\ 6 & 5.604371 & -1.068805 & -0.441924 \\ 1 & 5.957171 & -0.740201 & 0.546319 \\ 1 & 6.457634 & -1.051951 & -1.136922 \\ 1 & 5.254901 & -2.107443 & -0.349872 \\ 6 & 3.603075 & -0.556339 & -2.730861 \\ 1 & 3.217546 & -1.583912 & -2.656480 \\ 1 & 4.412176 & -0.544465 & -3.476877 \\ 1 & 2.788780 & 0.087701 & -3.094542 \\ 6 & 4.818215 & 1.821911 & -1.191553 \\ 1 & 4.003368 & 2.480316 & -1.527017 \\ 1 & 5.645737 & 1.903061 & -1.912622 \\ 1 & 5.172296 & 2.188558 & -0.217132\end{array}$


Table S4

\section{M06-2X/def2-SVP}

TS1

ENERGY (WITHOUT THE ZERO-POINT) = -6302.90104296 Hartree FREE ENERGY = -6302.269447 Hartree

\begin{tabular}{rrrr} 
Atomic & \multicolumn{3}{c}{ Coordinates (Angstroms) } \\
Number & X & Y & Z \\
32 & 0.661192 & -2.588895 & 0.420996 \\
32 & -1.086013 & -0.572381 & 0.601173 \\
14 & 0.937182 & 3.900687 & -0.386080 \\
14 & -5.946653 & 0.206513 & 1.489169 \\
14 & -2.924787 & -0.298331 & -2.626954 \\
7 & 2.162765 & -1.721146 & -0.627691 \\
7 & 1.947728 & -1.155145 & 1.472270 \\
6 & 2.687604 & -1.036392 & 0.395515 \\
6 & 2.570943 & -1.768571 & -2.040192 \\
6 & 2.024910 & -0.542149 & -2.785952 \\
1 & 2.466839 & 0.380504 & -2.382127 \\
1 & 0.933792 & -0.479515 & -2.676064 \\
1 & 2.275697 & -0.601787 & -3.856244 \\
6 & 1.969486 & -3.048686 & -2.635696 \\
1 & 2.360745 & -3.933295 & -2.110548 \\
1 & 2.238737 & -3.129205 & -3.698356 \\
1 & 0.874258 & -3.049942 & -2.559980
\end{tabular}




\begin{tabular}{|c|c|c|c|}
\hline 6 & 4.094153 & -1.847507 & -2.234295 \\
\hline 1 & 4.597251 & -0.892343 & -2.040489 \\
\hline 1 & 4.301839 & -2.126593 & -3.277793 \\
\hline 1 & 4.531309 & -2.616573 & -1.580090 \\
\hline 6 & 3.929926 & -0.210732 & 0.302083 \\
\hline 6 & 5.187830 & -0.725246 & 0.623380 \\
\hline 1 & 5.285078 & -1.768426 & 0.929772 \\
\hline 6 & 6.316260 & 0.088196 & 0.531811 \\
\hline 1 & 7.298016 & -0.317120 & 0.780700 \\
\hline 6 & 6.193039 & 1.414948 & 0.118049 \\
\hline 1 & 7.078163 & 2.048470 & 0.045697 \\
\hline 6 & 4.936170 & 1.930355 & -0.201537 \\
\hline 1 & 4.834199 & 2.969950 & -0.519831 \\
\hline 6 & 3.806719 & 1.119731 & -0.107018 \\
\hline 1 & 2.810171 & 1.507506 & -0.331961 \\
\hline 6 & 2.211411 & -0.739839 & 2.849830 \\
\hline 6 & 2.664669 & 0.724337 & 2.959710 \\
\hline 1 & 3.704696 & 0.868139 & 2.638318 \\
\hline 1 & 2.589277 & 1.048200 & 4.008706 \\
\hline 1 & 2.011491 & 1.366060 & 2.347737 \\
\hline 6 & 0.887447 & -0.885484 & 3.609791 \\
\hline 1 & 0.155696 & -0.138678 & 3.266630 \\
\hline 1 & 1.051322 & -0.731578 & 4.686052 \\
\hline 1 & 0.456518 & -1.886619 & 3.458744 \\
\hline 6 & 3.250792 & -1.669228 & 3.491566 \\
\hline 1 & 2.914203 & -2.714892 & 3.430722 \\
\hline
\end{tabular}




$\begin{array}{lrrr}1 & 3.398548 & -1.409887 & 4.550737 \\ 1 & 4.220034 & -1.580729 & 2.981538 \\ 6 & -0.708131 & -1.869389 & -1.110974 \\ 6 & -1.625004 & -1.160985 & -1.587175 \\ 6 & -2.994823 & 1.514580 & -2.162029 \\ 1 & -3.281596 & 1.637037 & -1.107320 \\ 1 & -3.733837 & 2.032405 & -2.793235 \\ 1 & -2.012958 & 1.989638 & -2.304856 \\ 6 & -4.561881 & -1.164201 & -2.329097 \\ 1 & -4.506997 & -2.218731 & -2.635758 \\ 1 & -5.364510 & -0.676798 & -2.903894 \\ 1 & -4.816033 & -1.126293 & -1.260156 \\ 6 & -2.370506 & -0.505554 & -4.410867 \\ 1 & -1.386508 & -0.039919 & -4.568067 \\ 1 & -3.089487 & -0.030481 & -5.095721 \\ 1 & -2.293020 & -1.569835 & -4.676245 \\ 6 & -2.957038 & -0.275145 & 0.946723 \\ 6 & -4.138437 & -0.101315 & 1.214758 \\ 6 & -6.178635 & 0.959592 & 3.193053 \\ 1 & -5.814360 & 0.276734 & 3.973985 \\ 1 & -7.243014 & 1.164882 & 3.382926 \\ 1 & -5.624185 & 1.905057 & 3.279964 \\ 6 & -6.523743 & 1.393664 & 0.150762 \\ 1 & -5.979559 & 2.347243 & 0.216916 \\ 1 & -7.599875 & 1.602035 & 0.250061 \\ 1 & -6.345461 & 0.967746 & -0.847763\end{array}$




$\begin{array}{lrrc}6 & -6.852413 & -1.433391 & 1.356070 \\ 1 & -6.706729 & -1.885195 & 0.364092 \\ 1 & -7.932337 & -1.289548 & 1.512304 \\ 1 & -6.485774 & -2.143318 & 2.111415 \\ 6 & -0.373277 & 1.167084 & 0.142174 \\ 6 & 0.189343 & 2.236920 & -0.058819 \\ 6 & 2.100358 & 4.330727 & 1.026182 \\ 1 & 1.547969 & 4.372907 & 1.976583 \\ 1 & 2.565346 & 5.314042 & 0.856329 \\ 1 & 2.900433 & 3.583569 & 1.134522 \\ 6 & -0.459409 & 5.151594 & -0.490395 \\ 1 & -1.159722 & 4.884548 & -1.295185 \\ 1 & -0.066722 & 6.159664 & -0.692330 \\ 1 & -1.022514 & 5.182183 & 0.453495 \\ 6 & 1.859556 & 3.829082 & -2.024479 \\ 1 & 2.688803 & 3.106786 & -2.002989 \\ 1 & 2.274459 & 4.818394 & -2.271411 \\ 1 & 1.176843 & 3.532362 & -2.834481\end{array}$


Table S5

\section{M06-2X/def2-SVP}

1

ENERGY (WITHOUT THE ZERO-POINT) $=-6302.95180253$ Hartree FREE ENERGY = -6302.325528 Hartree

\begin{tabular}{rrrr} 
Atomic & \multicolumn{3}{c}{ Coordinates (Angstroms) } \\
Number & X & Y & $Z$ \\
32 & 0.169661 & 0.083606 & -2.316179 \\
32 & 1.272147 & 0.152767 & -0.047307 \\
14 & -1.735168 & -1.164645 & 3.739399 \\
14 & 2.300048 & 4.985112 & 0.587895 \\
14 & 5.041735 & -3.112513 & -0.075834 \\
7 & -1.195893 & -1.213453 & -1.615681 \\
7 & -1.471334 & 0.928662 & -1.506128 \\
6 & -2.051419 & -0.250297 & -1.294610 \\
6 & -1.274556 & -2.674439 & -1.552523 \\
6 & -2.106964 & -3.171473 & -0.364655 \\
1 & -3.184618 & -3.022775 & -0.515107 \\
1 & -1.798780 & -2.649971 & 0.553632 \\
1 & -1.931191 & -4.249232 & -0.230977 \\
6 & 0.167935 & -3.161023 & -1.368740 \\
1 & 0.827744 & -2.746551 & -2.149757 \\
1 & 0.217214 & -4.257454 & -1.434932 \\
1 & 0.558910 & -2.854244 & -0.386443
\end{tabular}




$\begin{array}{llll}6 & -1.840991 & -3.219406 & -2.869132 \\ 1 & -2.874416 & -2.874012 & -3.017152 \\ 1 & -1.844836 & -4.319452 & -2.859419 \\ 1 & -1.232554 & -2.878475 & -3.720031 \\ 6 & -3.458887 & -0.463158 & -0.848643 \\ 6 & -4.438289 & -0.771263 & -1.797582 \\ 1 & -4.166774 & -0.829338 & -2.853607 \\ 6 & -5.755428 & -0.984535 & -1.395805 \\ 1 & -6.518684 & -1.219022 & -2.139107 \\ 6 & -6.097354 & -0.894719 & -0.046146 \\ 1 & -7.128349 & -1.064247 & 0.267740 \\ 6 & -5.120307 & -0.587896 & 0.901142 \\ 1 & -5.383109 & -0.524601 & 1.959025 \\ 6 & -3.802735 & -0.371193 & 0.501509 \\ 1 & -3.018992 & -0.151100 & 1.228384 \\ 6 & -1.955977 & 2.299991 & -1.320059 \\ 6 & -1.965841 & 2.663114 & 0.170298 \\ 1 & -2.664841 & 2.024231 & 0.727495 \\ 1 & -2.283790 & 3.708937 & 0.300770 \\ 1 & -0.961129 & 2.547073 & 0.603961 \\ 6 & -0.948561 & 3.199534 & -2.043471 \\ 1 & 0.061500 & 3.053803 & -1.626246 \\ 1 & -1.221045 & 4.257257 & -1.915702 \\ 1 & -0.927113 & 2.973338 & -3.120344 \\ 6 & -3.347609 & 2.512670 & -1.929889 \\ 1 & -3.370440 & 2.156083 & -2.970764\end{array}$




$\begin{array}{rrrr}1 & -3.586799 & 3.586303 & -1.927064 \\ 1 & -4.131054 & 1.992763 & -1.362541 \\ 6 & 2.793418 & -1.020339 & 0.086893 \\ 6 & 3.697398 & -1.842293 & 0.032621 \\ 6 & 5.566491 & -3.590448 & 1.662745 \\ 1 & 5.942411 & -2.713691 & 2.209579 \\ 1 & 6.364508 & -4.347690 & 1.631996 \\ 1 & 4.717490 & -4.004874 & 2.225372 \\ 6 & 6.484278 & -2.372055 & -1.022220 \\ 1 & 6.170954 & -2.061092 & -2.029230 \\ 1 & 7.298884 & -3.104985 & -1.124472 \\ 1 & 6.877961 & -1.488015 & -0.500099 \\ 6 & 4.344115 & -4.601281 & -0.987039 \\ 1 & 3.482032 & -5.017707 & -0.445394 \\ 1 & 5.103585 & -5.391544 & -1.087753 \\ 1 & 4.008438 & -4.315570 & -1.994731 \\ 6 & 1.837115 & 1.954268 & 0.346082 \\ 6 & 2.054715 & 3.153429 & 0.454673 \\ 6 & 0.663040 & 5.734133 & 1.126385 \\ 1 & -0.120524 & 5.518257 & 0.385112 \\ 1 & 0.747464 & 6.826523 & 1.231174 \\ 1 & 0.342204 & 5.317077 & 2.092231 \\ 6 & 3.644025 & 5.335010 & 1.850160 \\ 1 & 3.364772 & 4.939440 & 2.837304 \\ 1 & 3.812846 & 6.418276 & 1.946351 \\ 1 & 4.590664 & 4.863279 & 1.549435\end{array}$




$\begin{array}{lrrr}6 & 2.792448 & 5.632013 & -1.105358 \\ 1 & 3.725617 & 5.160229 & -1.445756 \\ 1 & 2.946189 & 6.721398 & -1.076963 \\ 1 & 2.010197 & 5.413665 & -1.847248 \\ 6 & 0.079462 & -0.354488 & 1.391488 \\ 6 & -0.685632 & -0.690883 & 2.286482 \\ 6 & -2.836979 & 0.298211 & 4.172363 \\ 1 & -2.222470 & 1.175360 & 4.423048 \\ 1 & -3.465168 & 0.058352 & 5.043937 \\ 1 & -3.500137 & 0.580097 & 3.341156 \\ 6 & -0.593556 & -1.557155 & 5.175902 \\ 1 & 0.074761 & -2.392456 & 4.922038 \\ 1 & -1.174394 & -1.836616 & 6.068100 \\ 1 & 0.029909 & -0.686854 & 5.425947 \\ 6 & -2.771492 & -2.671850 & 3.304692 \\ 1 & -3.446719 & -2.475608 & 2.458533 \\ 1 & -3.382536 & -2.967800 & 4.171562 \\ 1 & -2.126869 & -3.521628 & 3.035336\end{array}$


Table S6

\section{M06-2X/def2-SVP}

$\mathrm{L}_{2} \mathrm{Ge}-\mathrm{CCTMS}\left(\mathrm{L}_{2}=\mathrm{PhC}(\mathrm{NCy})_{2}\right)$

ENERGY (WITHOUT THE ZERO-POINT) $=-3410.66588887$ Hartree

FREE ENERGY $=-3410.179423$ Hartree

\begin{tabular}{rrrr} 
Atomic & \multicolumn{3}{c}{ Coordinates (Angstroms) } \\
Number & $\mathrm{X}$ & $\mathrm{Y}$ & $\mathrm{Z}$ \\
\hline 32 & -0.006128 & -0.618702 & -1.949875 \\
14 & 3.725670 & -2.316807 & 0.994580 \\
7 & -0.113324 & 1.178742 & -1.023308 \\
7 & -1.464269 & -0.449428 & -0.524588 \\
6 & 1.367122 & -1.312147 & -0.699311 \\
6 & 2.304056 & -1.744044 & -0.035527 \\
6 & 3.468669 & -1.714417 & 2.757795 \\
1 & 2.535905 & -2.122039 & 3.174284 \\
1 & 3.402459 & -0.616725 & 2.787169 \\
1 & 4.302119 & -2.027273 & 3.404982 \\
6 & 5.301714 & -1.579488 & 0.281495 \\
1 & 5.449959 & -1.912217 & -0.756098 \\
1 & 6.179773 & -1.883285 & 0.871602 \\
1 & 5.249335 & -0.480744 & 0.282607 \\
6 & 3.802401 & -4.194220 & 0.957550 \\
1 & 3.928985 & -4.559989 & -0.071592 \\
1 & 2.875864 & -4.628025 & 1.361142
\end{tabular}




$\begin{array}{rrrr}1 & 4.646458 & -4.560565 & 1.561543 \\ 6 & -1.163390 & 0.817903 & -0.295708 \\ 6 & 0.785867 & 2.287286 & -0.773962 \\ 1 & 0.215829 & 3.236262 & -0.750550 \\ 6 & 1.516921 & 2.139969 & 0.567579 \\ 1 & 2.036597 & 1.166593 & 0.560861 \\ 1 & 0.779200 & 2.107452 & 1.385088 \\ 6 & 2.512659 & 3.277565 & 0.786440 \\ 1 & 1.964202 & 4.233388 & 0.859604 \\ 1 & 3.034957 & 3.142230 & 1.745511 \\ 6 & 3.515401 & 3.362920 & -0.364257 \\ 1 & 4.211343 & 4.201276 & -0.209871 \\ 1 & 4.124019 & 2.442070 & -0.379918 \\ 6 & 2.795580 & 3.506641 & -1.705115 \\ 1 & 3.519613 & 3.530987 & -2.532850 \\ 1 & 2.259882 & 4.471888 & -1.726860 \\ 6 & 1.794473 & 2.373123 & -1.920082 \\ 1 & 1.257283 & 2.493017 & -2.873596 \\ 1 & 2.334842 & 1.409205 & -1.968333 \\ 6 & -1.899891 & 1.727998 & 0.622379 \\ 6 & -2.353995 & 2.965701 & 0.155365 \\ 1 & -2.183032 & 3.238669 & -0.887811 \\ 6 & -3.030419 & 3.829369 & 1.014192 \\ 1 & -3.389816 & 4.791204 & 0.645567 \\ 6 & -3.248701 & 3.463549 & 2.342194 \\ 1 & -3.775077 & 4.142191 & 3.015057\end{array}$




$\begin{array}{lllc}6 & -2.795721 & 2.229982 & 2.811338 \\ 1 & -2.964079 & 1.945462 & 3.850892 \\ 6 & -2.124766 & 1.361236 & 1.953872 \\ 1 & -1.757516 & 0.397476 & 2.313495 \\ 6 & -2.660402 & -1.164928 & -0.144284 \\ 1 & -3.289696 & -0.508454 & 0.484590 \\ 6 & -2.289768 & -2.406741 & 0.672354 \\ 1 & -1.713048 & -2.102598 & 1.559600 \\ 1 & -1.611171 & -3.027198 & 0.060305 \\ 6 & -3.527113 & -3.213320 & 1.062624 \\ 1 & -3.232879 & -4.109164 & 1.629372 \\ 1 & -4.157443 & -2.606589 & 1.736450 \\ 6 & -4.344536 & -3.600239 & -0.169956 \\ 1 & -3.744305 & -4.277410 & -0.801846 \\ 1 & -5.246416 & -4.157457 & 0.124834 \\ 6 & -4.723250 & -2.363136 & -0.984673 \\ 1 & -5.393131 & -1.725034 & -0.382088 \\ 1 & -5.287549 & -2.649269 & -1.884670 \\ 6 & -3.483563 & -1.559114 & -1.376200 \\ 1 & -3.757682 & -0.651757 & -1.936395 \\ 1 & -2.841987 & -2.163707 & -2.041529\end{array}$


Table S7

\section{M06-2X/def2-SVP}

TS2

ENERGY (WITHOUT THE ZERO-POINT) $=-6457.56918870$ Hartree FREE ENERGY = -6456.862555 Hartree

\begin{tabular}{rrrc} 
Atomic & \multicolumn{3}{c}{ Coordinates (Angstroms) } \\
Number & $\mathrm{X}$ & $\mathrm{Y}$ & $\mathrm{Z}$ \\
\hline 32 & 1.156405 & 1.512979 & 1.435556 \\
32 & -0.007916 & 0.751651 & -1.308030 \\
14 & -1.612958 & -2.213667 & 3.267162 \\
14 & -4.709904 & -1.017212 & -1.222172 \\
14 & -3.142664 & 4.199563 & -0.059568 \\
7 & 1.111927 & -0.936974 & -0.777183 \\
7 & 2.380555 & 0.730096 & 0.105723 \\
6 & 0.194819 & -0.117643 & 1.929664 \\
6 & -0.477500 & -0.971090 & 2.497044 \\
6 & -1.774054 & -0.113311 & -1.033247 \\
6 & -2.937892 & -0.499495 & -1.098132 \\
6 & -0.704479 & 2.337403 & 0.156782 \\
6 & -1.618483 & 3.161059 & 0.101386 \\
6 & -0.607892 & -3.695804 & 3.840639 \\
1 & 0.168364 & -3.389948 & 4.557032 \\
1 & -0.114907 & -4.191018 & 2.990882 \\
1 & -1.259534 & -4.433315 & 4.333663
\end{tabular}




$\begin{array}{lrrr}6 & -2.457304 & -1.391669 & 4.730068 \\ 1 & -3.030215 & -0.513057 & 4.399303 \\ 1 & -1.717848 & -1.055389 & 5.471147 \\ 1 & -3.149664 & -2.091561 & 5.222303 \\ 6 & -2.891068 & -2.729056 & 1.985940 \\ 1 & -3.151752 & -1.859871 & 1.362660 \\ 1 & -3.803196 & -3.112818 & 2.468350 \\ 1 & -2.495913 & -3.512132 & 1.323010 \\ 6 & -4.802323 & -2.891385 & -1.344294 \\ 1 & -4.302974 & -3.369315 & -0.488901 \\ 1 & -4.320321 & -3.243668 & -2.268273 \\ 1 & -5.851831 & -3.223301 & -1.360260 \\ 6 & -5.443088 & -0.246340 & -2.773074 \\ 1 & -5.400687 & 0.851355 & -2.724205 \\ 1 & -6.494903 & -0.548199 & -2.892869 \\ 1 & -4.886989 & -0.569167 & -3.665395 \\ 6 & -5.649591 & -0.419892 & 0.294580 \\ 1 & -5.580733 & 0.672599 & 0.399167 \\ 1 & -5.256726 & -0.874576 & 1.214975 \\ 1 & -6.713739 & -0.687810 & 0.206561 \\ 6 & -4.273118 & 3.279837 & -1.246569 \\ 1 & -3.812968 & 3.199805 & -2.242547 \\ 1 & -5.248299 & 3.779809 & -1.348122 \\ 1 & -4.434071 & 2.257817 & -0.872318 \\ 6 & -3.944063 & 4.345261 & 1.633091 \\ 1 & -3.267420 & 4.838364 & 2.345936\end{array}$




$\begin{array}{lrrr}1 & -4.185123 & 3.347363 & 2.027815 \\ 1 & -4.873741 & 4.931584 & 1.576095 \\ 6 & -2.693070 & 5.894438 & -0.732388 \\ 1 & -2.191047 & 5.799476 & -1.706010 \\ 1 & -2.013520 & 6.419981 & -0.045952 \\ 1 & -3.593497 & 6.512476 & -0.867265 \\ 6 & 2.280818 & -0.536004 & -0.373875 \\ 6 & 0.819365 & -2.322388 & -1.150400 \\ 1 & 1.766024 & -2.887715 & -1.156239 \\ 6 & -0.099342 & -2.996367 & -0.129084 \\ 1 & -1.025209 & -2.406002 & -0.046972 \\ 1 & 0.389520 & -2.967288 & 0.856486 \\ 6 & -0.412976 & -4.432065 & -0.546670 \\ 1 & 0.514033 & -5.032583 & -0.533659 \\ 1 & -1.091629 & -4.896205 & 0.186223 \\ 6 & -1.021281 & -4.478953 & -1.946819 \\ 1 & -1.247117 & -5.515931 & -2.237770 \\ 1 & -1.977676 & -3.928660 & -1.938140 \\ 6 & -0.080869 & -3.831069 & -2.961953 \\ 1 & -0.521418 & -3.851491 & -3.969741 \\ 1 & 0.854702 & -4.415671 & -3.013717 \\ 6 & 0.239210 & -2.389375 & -2.567929 \\ 1 & 0.952798 & -1.938333 & -3.276675 \\ 1 & -0.687427 & -1.794080 & -2.607528 \\ 6 & 3.507565 & -1.388203 & -0.422927 \\ 6 & 4.033562 & -1.831675 & -1.640842\end{array}$




$\begin{array}{llll}1 & 3.532516 & -1.562031 & -2.573598 \\ 6 & 5.193843 & -2.602175 & -1.656701 \\ 1 & 5.608433 & -2.940226 & -2.607431 \\ 6 & 5.822616 & -2.943014 & -0.458155 \\ 1 & 6.728082 & -3.551439 & -0.472121 \\ 6 & 5.293370 & -2.510241 & 0.757066 \\ 1 & 5.780641 & -2.782499 & 1.694230 \\ 6 & 4.140432 & -1.726570 & 0.776080 \\ 1 & 3.713832 & -1.380847 & 1.720567 \\ 6 & 3.600658 & 1.514981 & -0.184063 \\ 1 & 4.223517 & 0.916332 & -0.869600 \\ 6 & 4.457765 & 1.827779 & 1.046818 \\ 1 & 4.746032 & 0.888574 & 1.543493 \\ 1 & 3.859029 & 2.409461 & 1.767594 \\ 6 & 5.697187 & 2.634501 & 0.653697 \\ 1 & 6.296013 & 2.859492 & 1.548679 \\ 1 & 6.334915 & 2.021252 & -0.007111 \\ 6 & 5.310385 & 3.922817 & -0.072541 \\ 1 & 4.739015 & 4.567221 & 0.617482 \\ 1 & 6.210220 & 4.486044 & -0.362189 \\ 6 & 4.450820 & 3.619022 & -1.298746 \\ 1 & 5.051850 & 3.049720 & -2.029474 \\ 1 & 4.145697 & 4.549449 & -1.799824 \\ 6 & 3.216609 & 2.801476 & -0.922515 \\ 1 & 2.624125 & 2.541823 & -1.812745 \\ 1 & 2.557287 & 3.395822 & -0.265126\end{array}$


Table S8

\section{M06-2X/def2-SVP}

Int

ENERGY (WITHOUT THE ZERO-POINT) $=-6457.63530978$ Hartree FREE ENERGY = -6456.929492 Hartree

\begin{tabular}{rrrr} 
Atomic & \multicolumn{3}{c}{ Coordinates (Angstroms) } \\
Number & X & Y & Z \\
32 & -1.392768 & -0.190885 & -2.278590 \\
32 & 0.423620 & 0.832686 & -0.930758 \\
14 & 1.082258 & -4.383351 & -0.979697 \\
14 & 5.016082 & -1.069825 & -0.444228 \\
14 & 0.928655 & 5.805280 & -0.648138 \\
7 & -0.405300 & 0.373031 & 0.768961 \\
7 & -2.353900 & -0.042312 & -0.440115 \\
6 & -0.574567 & -1.900772 & -1.680511 \\
6 & 0.018222 & -2.924770 & -1.353655 \\
6 & 2.198572 & 0.087490 & -0.867698 \\
6 & 3.320196 & -0.392244 & -0.776284 \\
6 & 0.670422 & 2.742229 & -0.788428 \\
6 & 0.762456 & 3.961232 & -0.746023 \\
6 & 1.456417 & -4.449170 & 0.864087 \\
1 & 0.528148 & -4.527286 & 1.450310 \\
1 & 1.989577 & -3.543325 & 1.186766 \\
1 & 2.084491 & -5.322512 & 1.099380
\end{tabular}




$\begin{array}{lrrr}6 & 0.204411 & -5.963415 & -1.496897 \\ 1 & -0.030123 & -5.943816 & -2.570985 \\ 1 & -0.739502 & -6.083792 & -0.945208 \\ 1 & 0.834523 & -6.843307 & -1.296096 \\ 6 & 2.668987 & -4.144553 & -1.960789 \\ 1 & 2.472778 & -4.236692 & -3.039067 \\ 1 & 3.444275 & -4.875100 & -1.682562 \\ 1 & 3.047071 & -3.127497 & -1.776221 \\ 6 & 4.832346 & -2.510997 & 0.750244 \\ 1 & 4.284234 & -3.341402 & 0.282217 \\ 1 & 4.284090 & -2.206166 & 1.655007 \\ 1 & 5.821879 & -2.881941 & 1.058678 \\ 6 & 6.024956 & 0.307329 & 0.339589 \\ 1 & 6.102684 & 1.165766 & -0.343091 \\ 1 & 7.042138 & -0.040525 & 0.575363 \\ 1 & 5.555431 & 0.656109 & 1.271150 \\ 6 & 5.804393 & -1.627369 & -2.052384 \\ 1 & 5.872199 & -0.791086 & -2.763075 \\ 1 & 5.222347 & -2.432080 & -2.523711 \\ 1 & 6.821858 & -2.003333 & -1.865132 \\ 6 & 1.032390 & 6.278242 & 1.167547 \\ 1 & 0.125327 & 5.964769 & 1.704832 \\ 1 & 1.140391 & 7.367607 & 1.281512 \\ 1 & 1.896606 & 5.794198 & 1.645431 \\ 6 & 2.495838 & 6.306666 & -1.551871 \\ 1 & 2.451057 & 6.003491 & -2.607834\end{array}$




$\begin{array}{lrrr}1 & 3.376033 & 5.829590 & -1.097281 \\ 1 & 2.633931 & 7.397802 & -1.511429 \\ 6 & -0.580236 & 6.585077 & -1.449261 \\ 1 & -1.499808 & 6.278255 & -0.930307 \\ 1 & -0.663974 & 6.277137 & -2.501538 \\ 1 & -0.514122 & 7.683058 & -1.414662 \\ 6 & -1.701307 & 0.041005 & 0.701953 \\ 6 & 0.352565 & 0.262976 & 2.028505 \\ 1 & -0.356230 & 0.355976 & 2.865097 \\ 6 & 1.020841 & -1.110569 & 2.143755 \\ 1 & 1.701977 & -1.241776 & 1.287930 \\ 1 & 0.251877 & -1.894192 & 2.057787 \\ 6 & 1.798219 & -1.241962 & 3.451731 \\ 1 & 1.095285 & -1.211434 & 4.303029 \\ 1 & 2.298908 & -2.222250 & 3.493753 \\ 6 & 2.812434 & -0.107722 & 3.599286 \\ 1 & 3.362913 & -0.201299 & 4.547491 \\ 1 & 3.556738 & -0.183331 & 2.786127 \\ 6 & 2.122492 & 1.253438 & 3.511155 \\ 1 & 2.856619 & 2.066725 & 3.610222 \\ 1 & 1.419415 & 1.357859 & 4.356240 \\ 6 & 1.363485 & 1.400413 & 2.191901 \\ 1 & 0.840888 & 2.367475 & 2.135243 \\ 1 & 2.090126 & 1.380806 & 1.363538 \\ 6 & -2.421160 & -0.266952 & 1.983457 \\ 6 & -2.913359 & 0.756961 & 2.796362\end{array}$




$\begin{array}{llrl}1 & -2.765707 & 1.798479 & 2.502389 \\ 6 & -3.584621 & 0.441609 & 3.976148 \\ 1 & -3.972256 & 1.240356 & 4.610014 \\ 6 & -3.759019 & -0.892058 & 4.347359 \\ 1 & -4.283622 & -1.135913 & 5.272230 \\ 6 & -3.266012 & -1.912751 & 3.535407 \\ 1 & -3.402894 & -2.955953 & 3.823380 \\ 6 & -2.600286 & -1.602952 & 2.349755 \\ 1 & -2.215541 & -2.392751 & 1.699317 \\ 6 & -3.805346 & -0.259518 & -0.481282 \\ 1 & -4.203251 & -0.236502 & 0.544728 \\ 6 & -4.153790 & -1.629461 & -1.069667 \\ 1 & -3.648868 & -2.415674 & -0.487357 \\ 1 & -3.756828 & -1.696116 & -2.098360 \\ 6 & -5.667344 & -1.841776 & -1.095041 \\ 1 & -5.900942 & -2.819543 & -1.541628 \\ 1 & -6.047697 & -1.868269 & -0.058634 \\ 6 & -6.369472 & -0.719487 & -1.859877 \\ 1 & -6.051811 & -0.750721 & -2.916366 \\ 1 & -7.459107 & -0.872109 & -1.851271 \\ 6 & -6.017828 & 0.647495 & -1.273421 \\ 1 & -6.411726 & 0.711281 & -0.243659 \\ 1 & -6.504869 & 1.450741 & -1.845977 \\ 6 & -4.504999 & 0.866476 & -1.249663 \\ 1 & -4.252609 & 1.831436 & -0.782711 \\ 1 & -4.116886 & 0.895809 & -2.281837\end{array}$


Table S9

\section{M06-2X/def2-SVP}

TS3

ENERGY (WITHOUT THE ZERO-POINT) $=-6457.5603996$ Hartree FREE ENERGY = -6456.871726 Hartree

\begin{tabular}{rrrc} 
Atomic & \multicolumn{3}{c}{ Coordinates (Angstroms) } \\
Number & $\mathrm{X}$ & $\mathrm{Y}$ & $\mathrm{Z}$ \\
32 & 1.734022 & 0.944243 & 0.159479 \\
32 & -1.358783 & -0.322371 & 0.518242 \\
32 & 0.492424 & 0.222128 & -1.879175 \\
14 & 6.083177 & 2.074266 & -2.018551 \\
14 & 2.703201 & -1.724564 & 4.273136 \\
14 & -6.201830 & -0.047819 & 1.594960 \\
14 & -3.300431 & -1.401341 & -4.046935 \\
7 & -0.516660 & 2.427867 & -1.039059 \\
7 & 0.903790 & 2.519127 & 0.809350 \\
7 & -0.555098 & -2.167016 & 0.580342 \\
7 & 0.859490 & -1.821293 & -1.276082 \\
6 & 3.476616 & 1.509712 & -0.488492 \\
6 & 4.516969 & 1.753087 & -1.086778 \\
6 & 2.140013 & -0.193738 & 1.667542 \\
6 & 2.387817 & -0.874068 & 2.654767 \\
6 & -3.267634 & -0.650303 & 0.920423 \\
6 & -4.446793 & -0.460241 & 1.204604
\end{tabular}




$\begin{array}{lrrl}6 & -1.740305 & -0.622256 & -1.499264 \\ 6 & -2.343048 & -0.949887 & -2.523046 \\ 6 & 7.366581 & 0.815741 & -1.469787 \\ 1 & 7.016289 & -0.208618 & -1.663329 \\ 1 & 7.564616 & 0.908614 & -0.392173 \\ 1 & 8.313720 & 0.964798 & -2.010112 \\ 6 & 5.717531 & 1.886704 & -3.853310 \\ 1 & 4.954972 & 2.612146 & -4.171997 \\ 1 & 5.338505 & 0.877857 & -4.074722 \\ 1 & 6.625243 & 2.053237 & -4.453036 \\ 6 & 6.668309 & 3.819275 & -1.640429 \\ 1 & 5.909895 & 4.556859 & -1.940339 \\ 1 & 7.600312 & 4.043010 & -2.181278 \\ 1 & 6.855997 & 3.941498 & -0.563845 \\ 6 & 2.385952 & -3.565563 & 4.067340 \\ 1 & 3.169498 & -4.037561 & 3.456850 \\ 1 & 1.420001 & -3.736893 & 3.567822 \\ 1 & 2.365134 & -4.067565 & 5.046603 \\ 6 & 4.486085 & -1.404351 & 4.768438 \\ 1 & 5.179239 & -1.793858 & 4.008976 \\ 1 & 4.715407 & -1.890803 & 5.728611 \\ 1 & 4.670241 & -0.325344 & 4.875697 \\ 6 & 1.532401 & -0.989801 & 5.547208 \\ 1 & 0.482245 & -1.181589 & 5.284409 \\ 1 & 1.672061 & 0.098774 & 5.625576 \\ 1 & 1.725360 & -1.432719 & 6.536422\end{array}$




$\begin{array}{lrrc}6 & -6.806971 & -1.155971 & 2.988444 \\ 1 & -6.188860 & -1.024154 & 3.888539 \\ 1 & -6.752599 & -2.213578 & 2.691057 \\ 1 & -7.850818 & -0.925031 & 3.249737 \\ 6 & -7.247273 & -0.309961 & 0.053466 \\ 1 & -6.879076 & 0.321024 & -0.769307 \\ 1 & -8.300594 & -0.053022 & 0.242322 \\ 1 & -7.200555 & -1.358586 & -0.275174 \\ 6 & -6.265838 & 1.762183 & 2.104520 \\ 1 & -5.877091 & 2.393755 & 1.291640 \\ 1 & -5.647848 & 1.941131 & 2.996553 \\ 1 & -7.296565 & 2.078067 & 2.325688 \\ 6 & -3.141639 & -3.251537 & -4.338617 \\ 1 & -2.092176 & -3.534857 & -4.502420 \\ 1 & -3.719939 & -3.551110 & -5.225916 \\ 1 & -3.521215 & -3.815555 & -3.474138 \\ 6 & -5.096922 & -0.949268 & -3.735987 \\ 1 & -5.224771 & 0.131166 & -3.578711 \\ 1 & -5.468171 & -1.467265 & -2.839698 \\ 1 & -5.718181 & -1.251395 & -4.593009 \\ 6 & -2.583707 & -0.472068 & -5.514566 \\ 1 & -1.514067 & -0.703067 & -5.630549 \\ 1 & -2.690047 & 0.614698 & -5.402801 \\ 1 & -3.102503 & -0.774697 & -6.437234 \\ 6 & -0.132775 & 2.998628 & 0.062080 \\ 6 & -1.606768 & 3.018928 & -1.815491\end{array}$




\begin{tabular}{lrrr}
1 & -1.600272 & 4.118212 & -1.696747 \\
6 & -1.413780 & 2.742345 & -3.307545 \\
1 & -1.441481 & 1.652229 & -3.469710 \\
1 & -0.414547 & 3.086842 & -3.616937 \\
6 & -2.511503 & 3.404776 & -4.140491 \\
1 & -2.439141 & 4.501827 & -4.039860 \\
1 & -2.363280 & 3.181661 & -5.208552 \\
6 & -3.897937 & 2.951231 & -3.681900 \\
1 & -4.000755 & 1.864638 & -3.857190 \\
1 & -4.685241 & 3.446684 & -4.270341 \\
6 & -4.094327 & 3.210594 & -2.187597 \\
1 & -5.082027 & 2.848330 & -1.862096 \\
1 & -4.078203 & 4.298842 & -1.999690 \\
6 & -2.994772 & 2.534332 & -1.369469 \\
1 & -3.135664 & 2.729940 & -0.297626 \\
1 & -3.057902 & 1.444902 & -1.507271 \\
6 & -0.813819 & 4.238131 & 0.584382 \\
6 & -0.361552 & 5.505030 & 0.202685 \\
1 & 0.489309 & 5.588308 & -0.477164 \\
6 & -0.990367 & 6.648933 & 0.690773 \\
1 & -0.633705 & 7.634777 & 0.388504 \\
6 & -2.071603 & 6.534061 & 1.565168 \\
1 & -2.562460 & 7.429993 & 1.948002 \\
6 & -2.524117 & 5.271985 & 1.947933 \\
1 & -3.370472 & 5.175639 & 2.629858 \\
6 & -1.897827 & 4.125605 & 1.458937 \\
\hline 6 & & & \\
1 & & & \\
1 & & &
\end{tabular}




$\begin{array}{rrrr}1 & -2.250380 & 3.134122 & 1.754203 \\ 6 & 1.255462 & 3.104291 & 2.113731 \\ 1 & 0.914297 & 4.150513 & 2.115989 \\ 6 & 2.772479 & 3.155390 & 2.323673 \\ 1 & 3.238187 & 3.691894 & 1.483205 \\ 1 & 3.189269 & 2.134161 & 2.320701 \\ 6 & 3.111293 & 3.815828 & 3.660119 \\ 1 & 4.202209 & 3.836183 & 3.800987 \\ 1 & 2.774016 & 4.867165 & 3.647470 \\ 6 & 2.431932 & 3.086680 & 4.818660 \\ 1 & 2.681761 & 3.565511 & 5.777641 \\ 1 & 2.821068 & 2.053232 & 4.864297 \\ 6 & 0.917206 & 3.042163 & 4.617733 \\ 1 & 0.431693 & 2.497760 & 5.442458 \\ 1 & 0.519937 & 4.072491 & 4.644682 \\ 6 & 0.556058 & 2.394823 & 3.280860 \\ 1 & 0.853542 & 1.334293 & 3.285811 \\ 1 & -0.531615 & 2.415705 & 3.118755 \\ 6 & 0.384317 & -2.557426 & -0.279039 \\ 6 & -0.952829 & -3.030063 & 1.704199 \\ 1 & -0.151816 & -3.763315 & 1.890216 \\ 6 & -1.095689 & -2.226037 & 3.002934 \\ 1 & -1.883821 & -1.462605 & 2.892473 \\ 1 & -0.149478 & -1.693295 & 3.191531 \\ 6 & -1.484114 & -3.134020 & 4.170371 \\ 1 & -0.673026 & -3.857427 & 4.368479\end{array}$




$\begin{array}{lrrr}1 & -1.602068 & -2.535925 & 5.087191 \\ 6 & -2.771167 & -3.898414 & 3.858748 \\ 1 & -3.044064 & -4.551303 & 4.701626 \\ 1 & -3.594973 & -3.175058 & 3.728917 \\ 6 & -2.618516 & -4.714368 & 2.575469 \\ 1 & -3.551630 & -5.250973 & 2.347218 \\ 1 & -1.840382 & -5.484149 & 2.725835 \\ 6 & -2.228558 & -3.822999 & 1.396248 \\ 1 & -2.064428 & -4.429028 & 0.492743 \\ 1 & -3.041988 & -3.116077 & 1.170831 \\ 6 & 0.949595 & -3.944038 & -0.097537 \\ 6 & 0.324106 & -5.045558 & -0.684977 \\ 1 & -0.586611 & -4.899417 & -1.270245 \\ 6 & 0.871747 & -6.319679 & -0.533259 \\ 1 & 0.382602 & -7.178937 & -0.994460 \\ 6 & 2.042525 & -6.494666 & 0.203640 \\ 1 & 2.469891 & -7.491459 & 0.321478 \\ 6 & 2.668586 & -5.392308 & 0.787252 \\ 1 & 3.587185 & -5.527069 & 1.361003 \\ 6 & 2.126599 & -4.117252 & 0.635903 \\ 1 & 2.604016 & -3.241435 & 1.084167 \\ 6 & 1.843763 & -2.390484 & -2.207451 \\ 1 & 2.131483 & -3.398849 & -1.880488 \\ 6 & 3.145101 & -1.578154 & -2.222847 \\ 1 & 3.544021 & -1.511651 & -1.197012 \\ 1 & 2.959745 & -0.539867 & -2.554310\end{array}$




$\begin{array}{llll}6 & 4.165740 & -2.210656 & -3.168417 \\ 1 & 5.086668 & -1.607895 & -3.184854 \\ 1 & 4.446958 & -3.206801 & -2.783878 \\ 6 & 3.591011 & -2.359412 & -4.577535 \\ 1 & 3.379808 & -1.356341 & -4.987877 \\ 1 & 4.329226 & -2.827216 & -5.246180 \\ 6 & 2.297778 & -3.175961 & -4.559238 \\ 1 & 2.525611 & -4.202535 & -4.222190 \\ 1 & 1.881952 & -3.262947 & -5.574246 \\ 6 & 1.263028 & -2.558526 & -3.617558 \\ 1 & 0.362690 & -3.189382 & -3.549852 \\ 1 & 0.940431 & -1.579197 & -4.011827\end{array}$


Table S10

\section{M06-2X/def2-SVP}

2

ENERGY (WITHOUT THE ZERO-POINT) $=-6457.6361779$ Hartree FREE ENERGY $=-6456.941064$ Hartree

\begin{tabular}{rrrr} 
Atomic & \multicolumn{3}{c}{ Coordinates (Angstroms) } \\
Number & $\mathrm{X}$ & $\mathrm{Y}$ & $\mathrm{Z}$ \\
\hline 32 & -1.743085 & 0.442756 & 0.331147 \\
32 & 1.743057 & -0.442753 & 0.331398 \\
32 & -0.000292 & -0.000683 & 2.010056 \\
14 & -6.159266 & 0.748929 & 2.658434 \\
14 & -2.672849 & -1.937646 & -3.956893 \\
14 & 2.673395 & 1.938990 & -3.955749 \\
14 & 6.159330 & -0.750029 & 2.658434 \\
7 & 0.266904 & 2.300532 & 1.400770 \\
7 & -1.313042 & 2.194783 & -0.304901 \\
7 & 1.313094 & -2.194627 & -0.305315 \\
7 & -0.267324 & -2.300809 & 1.399924 \\
6 & -3.512940 & 0.635229 & 1.098443 \\
6 & -4.573780 & 0.693919 & 1.705265 \\
6 & -2.059371 & -0.641391 & -1.242243 \\
6 & -2.288599 & -1.260420 & -2.273377 \\
6 & 2.059395 & 0.641946 & -1.241589 \\
6 & 2.288752 & 1.261255 & -2.272527
\end{tabular}




$\begin{array}{lrrr}6 & 3.512874 & -0.635485 & 1.098690 \\ 6 & 4.573729 & -0.694448 & 1.705458 \\ 6 & -7.290759 & -0.605756 & 2.013849 \\ 1 & -6.821932 & -1.594102 & 2.126508 \\ 1 & -7.509580 & -0.452842 & 0.947131 \\ 1 & -8.242907 & -0.610528 & 2.565825 \\ 6 & -5.760832 & 0.470418 & 4.473432 \\ 1 & -5.075979 & 1.246837 & 4.843993 \\ 1 & -5.276074 & -0.506142 & 4.620492 \\ 1 & -6.676587 & 0.495853 & 5.083479 \\ 6 & -6.950138 & 2.435120 & 2.408180 \\ 1 & -6.286594 & 3.233600 & 2.770653 \\ 1 & -7.902497 & 2.503029 & 2.955668 \\ 1 & -7.151316 & 2.615870 & 1.342211 \\ 6 & -2.378364 & -3.793269 & -4.009819 \\ 1 & -3.038707 & -4.309700 & -3.296611 \\ 1 & -1.336532 & -4.055230 & -3.771461 \\ 1 & -2.605376 & -4.174203 & -5.017454 \\ 6 & -4.479409 & -1.574412 & -4.320508 \\ 1 & -5.130195 & -2.075607 & -3.589381 \\ 1 & -4.752740 & -1.927404 & -5.326546 \\ 1 & -4.679940 & -0.494309 & -4.267979 \\ 6 & -1.547262 & -1.049004 & -5.167964 \\ 1 & -0.496959 & -1.158917 & -4.861777 \\ 1 & -1.777306 & 0.027108 & -5.198171 \\ 1 & -1.659538 & -1.453927 & -6.185306\end{array}$




\begin{tabular}{|c|c|c|c|}
\hline 6 & 1.548114 & 1.050884 & -5.167483 \\
\hline 1 & 0.497668 & 1.161330 & -4.861972 \\
\hline 1 & 1.777626 & -0.025342 & -5.197615 \\
\hline 1 & 1.661262 & 1.455819 & -6.184722 \\
\hline 6 & 4.480074 & 1.575833 & -4.318894 \\
\hline 1 & 5.130534 & 2.076508 & -3.587116 \\
\hline 1 & 4.753904 & 1.929489 & -5.324558 \\
\hline 1 & 4.680531 & 0.495687 & -4.266977 \\
\hline 6 & 2.378895 & 3.794620 & -4.008244 \\
\hline 1 & 3.039166 & 4.310998 & -3.294941 \\
\hline 1 & 1.337030 & 4.056451 & -3.769873 \\
\hline 1 & 2.605868 & 4.175748 & -5.015817 \\
\hline 6 & 6.949749 & -2.436328 & 2.407489 \\
\hline 1 & 6.285491 & -3.234734 & 2.768824 \\
\hline 1 & 7.901604 & -2.505121 & 2.955746 \\
\hline 1 & 7.151738 & -2.616412 & 1.341562 \\
\hline 6 & 7.290785 & 0.604863 & 2.014259 \\
\hline 1 & 6.821424 & 1.593011 & 2.126496 \\
\hline 1 & 7.510257 & 0.451916 & 0.947680 \\
\hline 1 & 8.242608 & 0.610177 & 2.566776 \\
\hline 6 & 5.761214 & -0.472201 & 4.473609 \\
\hline 1 & 5.076343 & -1.248693 & 4.843979 \\
\hline 1 & 5.276629 & 0.504365 & 4.621172 \\
\hline 1 & 6.677095 & -0.498017 & 5.083456 \\
\hline 6 & -0.356342 & 2.856466 & 0.406386 \\
\hline 6 & 1.194863 & 3.059803 & 2.232541 \\
\hline
\end{tabular}




$\begin{array}{rrrr}1 & 1.359833 & 4.066450 & 1.811664 \\ 6 & 0.623311 & 3.252385 & 3.641526 \\ 1 & 0.424630 & 2.259465 & 4.079945 \\ 1 & -0.345388 & 3.772365 & 3.570076 \\ 6 & 1.596077 & 4.032030 & 4.526548 \\ 1 & 1.716915 & 5.052023 & 4.120662 \\ 1 & 1.179426 & 4.143749 & 5.538919 \\ 6 & 2.965073 & 3.352991 & 4.582141 \\ 1 & 2.858078 & 2.366134 & 5.065506 \\ 1 & 3.659123 & 3.939526 & 5.203325 \\ 6 & 3.534593 & 3.158392 & 3.176603 \\ 1 & 4.501422 & 2.632363 & 3.216651 \\ 1 & 3.729167 & 4.145901 & 2.722121 \\ 6 & 2.560864 & 2.375452 & 2.298050 \\ 1 & 2.964120 & 2.255236 & 1.278581 \\ 1 & 2.441300 & 1.363130 & 2.719921 \\ 6 & -0.068798 & 4.279101 & 0.010916 \\ 6 & -0.864096 & 5.316235 & 0.506228 \\ 1 & -1.705039 & 5.081751 & 1.163027 \\ 6 & -0.586146 & 6.635815 & 0.154738 \\ 1 & -1.208074 & 7.443484 & 0.543576 \\ 6 & 0.483635 & 6.922387 & -0.693884 \\ 1 & 0.699929 & 7.955658 & -0.969639 \\ 6 & 1.278138 & 5.887492 & -1.185593 \\ 1 & 2.116309 & 6.110983 & -1.847731 \\ 6 & 1.007324 & 4.565342 & -0.832209\end{array}$




$\begin{array}{lrrr}1 & 1.629940 & 3.744689 & -1.199699 \\ 6 & -1.894102 & 2.776494 & -1.524512 \\ 1 & -1.813889 & 3.871784 & -1.448814 \\ 6 & -3.389723 & 2.476557 & -1.661182 \\ 1 & -3.914825 & 2.819498 & -0.756949 \\ 1 & -3.554947 & 1.389030 & -1.731413 \\ 6 & -3.957069 & 3.141746 & -2.915740 \\ 1 & -5.026646 & 2.902746 & -3.013613 \\ 1 & -3.887010 & 4.239100 & -2.815083 \\ 6 & -3.193371 & 2.706611 & -4.166316 \\ 1 & -3.610777 & 3.189781 & -5.062687 \\ 1 & -3.320659 & 1.617139 & -4.300511 \\ 6 & -1.703515 & 3.017928 & -4.029737 \\ 1 & -1.154330 & 2.692327 & -4.927963 \\ 1 & -1.567843 & 4.112559 & -3.962064 \\ 6 & -1.119433 & 2.359879 & -2.780767 \\ 1 & -1.150830 & 1.260394 & -2.874417 \\ 1 & -0.060815 & 2.631098 & -2.646529 \\ 6 & 0.356247 & -2.856487 & 0.405553 \\ 6 & 1.894229 & -2.775919 & -1.525106 \\ 1 & 1.813975 & -3.871235 & -1.449833 \\ 6 & 1.119624 & -2.358764 & -2.781224 \\ 1 & 1.151044 & -1.259234 & -2.874383 \\ 1 & 0.060989 & -2.630014 & -2.647160 \\ 6 & 1.703724 & -3.016277 & -4.030457 \\ 1 & 1.568034 & -4.110937 & -3.963281\end{array}$




$\begin{array}{llll}1 & 1.154560 & -2.690262 & -4.928549 \\ 6 & 3.193589 & -2.704928 & -4.166838 \\ 1 & 3.611014 & -3.187719 & -5.063405 \\ 1 & 3.320915 & -1.615403 & -4.300560 \\ 6 & 3.957228 & -3.140636 & -2.916421 \\ 1 & 5.026818 & -2.901625 & -3.014146 \\ 1 & 3.887137 & -4.238032 & -2.816255 \\ 6 & 3.389871 & -2.475989 & -1.661579 \\ 1 & 3.914928 & -2.819382 & -0.757491 \\ 1 & 3.555161 & -1.388443 & -1.731290 \\ 6 & 0.068833 & -4.279028 & 0.009660 \\ 6 & 0.864076 & -5.316246 & 0.504877 \\ 1 & 1.704839 & -5.081901 & 1.161953 \\ 6 & 0.586305 & -6.635746 & 0.152931 \\ 1 & 1.208191 & -7.443487 & 0.541686 \\ 6 & -0.483222 & -6.922139 & -0.696063 \\ 1 & -0.699377 & -7.955340 & -0.972186 \\ 6 & -1.277664 & -5.887149 & -1.187694 \\ 1 & -2.115621 & -6.110496 & -1.850150 \\ 6 & -1.007042 & -4.565090 & -0.833843 \\ 1 & -1.629602 & -3.744362 & -1.201256 \\ 6 & -1.195377 & -3.060368 & 2.231363 \\ 1 & -1.360242 & -4.066873 & 1.810124 \\ 6 & -2.561443 & -2.376144 & 2.296918 \\ 1 & -2.964577 & -2.255612 & 1.277440 \\ 1 & -2.442055 & -1.363947 & 2.719143\end{array}$




$\begin{array}{lrrr}6 & -3.535229 & -3.159502 & 3.175042 \\ 1 & -4.502110 & -2.633569 & 3.215146 \\ 1 & -3.729653 & -4.146846 & 2.720138 \\ 6 & -2.965903 & -3.354610 & 4.580587 \\ 1 & -2.859063 & -2.367941 & 5.064368 \\ 1 & -3.659990 & -3.941460 & 5.201431 \\ 6 & -1.596837 & -4.033500 & 4.524931 \\ 1 & -1.717514 & -5.053348 & 4.118631 \\ 1 & -1.180323 & -4.145567 & 5.537320 \\ 6 & -0.624010 & -3.253419 & 3.640359 \\ 1 & 0.344749 & -3.773280 & 3.568863 \\ 1 & -0.425497 & -2.260651 & 4.079199\end{array}$

Table S1 1

M06-2X/def2-SVP

$\mathrm{R}^{1} \mathrm{Li}\left(\mathrm{R}^{1}=\mathrm{PhC}(\mathrm{N} t \mathrm{Bu})_{2}\right)$

ENERGY (WITHOUT THE ZERO-POINT) $=-701.674598950$ Hartree

FREE ENERGY $=-701.366189$ Hartree

\begin{tabular}{lrrr} 
& \multicolumn{2}{c}{ Atomic } & \multicolumn{3}{c}{ Coordinates (Angstroms) } \\
& Number & X & \multicolumn{2}{c}{ Y } & Z \\
- & & & \\
C & 0.01330 & 0.22504 & 1.41814 \\
C & -0.80977 & 1.40426 & 1.86903
\end{tabular}




$\begin{array}{lrcc}\mathrm{C} & -2.34164 & 3.59677 & 2.70543 \\ \mathrm{C} & -1.82440 & 1.23896 & 2.81652 \\ \mathrm{C} & -0.56943 & 2.67756 & 1.34412 \\ \mathrm{C} & -1.33169 & 3.76835 & 1.75851 \\ \mathrm{C} & -2.58578 & 2.32916 & 3.23421 \\ \mathrm{H} & -2.02750 & 0.24326 & 3.21553 \\ \mathrm{H} & 0.23351 & 2.81440 & 0.61721 \\ \mathrm{H} & -1.13347 & 4.75822 & 1.34429 \\ \mathrm{H} & -3.37710 & 2.18811 & 3.97219 \\ \mathrm{H} & -2.93796 & 4.45036 & 3.03118 \\ \mathrm{~N} & -0.30243 & -0.48861 & 0.33972 \\ \mathrm{~N} & 1.10277 & -0.17005 & 2.07309 \\ \mathrm{Li} & 1.22985 & -1.51075 & 0.75282 \\ \mathrm{C} & -1.35256 & -0.24701 & -0.64124 \\ \mathrm{C} & 1.64065 & 0.32341 & 3.33442 \\ \mathrm{C} & 1.73842 & 1.85467 & 3.46455 \\ \mathrm{H} & 2.16587 & 2.28977 & 2.54850 \\ \mathrm{H} & 0.76533 & 2.32606 & 3.65107 \\ \mathrm{H} & 2.39857 & 2.10988 & 4.30763 \\ \mathrm{C} & 0.85066 & -0.23561 & 4.52944 \\ \mathrm{H} & 1.34157 & 0.02197 & 5.48100 \\ \mathrm{H} & 0.78226 & -1.33147 & 4.45692 \\ \mathrm{H} & -0.16739 & 0.17612 & 4.55240 \\ \mathrm{C} & 3.07031 & -0.23450 & 3.40642 \\ \mathrm{H} & 3.66854 & 0.15728 & 2.56990 \\ \mathrm{H} & 3.05039 & -1.33304 & 3.33424\end{array}$




$\begin{array}{lrrr}\mathrm{H} & 3.56335 & 0.04107 & 4.35046 \\ \mathrm{C} & -1.47537 & -1.56021 & -1.42918 \\ \mathrm{H} & -0.50447 & -1.82343 & -1.87691 \\ \mathrm{H} & -2.21755 & -1.47743 & -2.23697 \\ \mathrm{H} & -1.77875 & -2.37528 & -0.75487 \\ \mathrm{C} & -2.74372 & 0.07407 & -0.06447 \\ \mathrm{H} & -2.81563 & 1.09978 & 0.31816 \\ \mathrm{H} & -2.98853 & -0.62059 & 0.75322 \\ \mathrm{H} & -3.50332 & -0.04040 & -0.85291 \\ \mathrm{C} & -0.94569 & 0.86250 & -1.62507 \\ \mathrm{H} & 0.04276 & 0.64158 & -2.05523 \\ \mathrm{H} & -0.89527 & 1.83558 & -1.11806 \\ \mathrm{H} & -1.67395 & 0.94634 & -2.44691 \\ ----------------------------------------------------------\end{array}$

\section{Table S12}

\section{M06-2X/def2-SVP}

$\mathrm{R}^{2} \mathrm{Li} \quad\left(\mathrm{R}^{2}=\mathrm{PhC}(\mathrm{NCy})_{2}\right)$

ENERGY (WITHOUT THE ZERO-POINT) $=-856.360272390$ Hartree FREE ENERGY $=-855.978910$ Hartree

\begin{tabular}{lrrr} 
& \multicolumn{2}{c}{ Atomic } & \multicolumn{3}{c}{ Coordinates (Angstroms) } \\
& Number & X & \multicolumn{2}{c}{ Y } & Z \\
- & & & \\
C & 0.36658 & 0.01024 & 1.29956 \\
C & -0.61850 & 0.99005 & 1.88431
\end{tabular}




$\begin{array}{lccc}\mathrm{C} & -2.43470 & 2.80438 & 3.00487 \\ \mathrm{C} & -1.66042 & 0.53559 & 2.69918 \\ \mathrm{C} & -0.49389 & 2.36004 & 1.63499 \\ \mathrm{C} & -1.39940 & 3.26321 & 2.19130 \\ \mathrm{C} & -2.56314 & 1.43873 & 3.25834 \\ \mathrm{H} & -1.75782 & -0.53476 & 2.89366 \\ \mathrm{H} & 0.32043 & 2.71743 & 1.00069 \\ \mathrm{H} & -1.29483 & 4.33052 & 1.98982 \\ \mathrm{H} & -3.37104 & 1.07488 & 3.89533 \\ \mathrm{H} & -3.14171 & 3.51112 & 3.44194 \\ \mathrm{~N} & 0.07323 & -0.70596 & 0.21870 \\ \mathrm{~N} & 1.55529 & -0.18243 & 1.85778 \\ \mathrm{Li} & 1.79276 & -1.44150 & 0.46367 \\ \mathrm{C} & -1.14260 & -0.50050 & -0.53555 \\ \mathrm{H} & -1.96995 & -0.18368 & 0.12383 \\ \mathrm{C} & -1.56083 & -1.81762 & -1.20636 \\ \mathrm{H} & -2.55862 & -1.68360 & -1.65825 \\ \mathrm{H} & -1.65302 & -2.59795 & -0.43539 \\ \mathrm{C} & -0.97066 & 0.58805 & -1.61377 \\ \mathrm{H} & -0.65396 & 1.52537 & -1.13053 \\ \mathrm{H} & -1.95087 & 0.78376 & -2.08334 \\ \mathrm{C} & -0.57910 & -2.24956 & -2.29645 \\ \mathrm{H} & 0.39813 & -2.47708 & -1.83575 \\ \mathrm{H} & -0.92276 & -3.18070 & -2.77273 \\ \mathrm{C} & 0.02815 & 0.16759 & -2.69086 \\ \mathrm{H} & 1.02088 & 0.04313 & -2.22592\end{array}$




$\begin{array}{lccc}\mathrm{H} & 0.12979 & 0.95723 & -3.45088 \\ \mathrm{C} & -0.39414 & -1.14992 & -3.34297 \\ \mathrm{H} & 0.33887 & -1.45969 & -4.10400 \\ \mathrm{H} & -1.35128 & -0.99631 & -3.87218 \\ \mathrm{C} & 1.96570 & 0.47031 & 3.07519 \\ \mathrm{H} & 1.10802 & 0.95494 & 3.58132 \\ \mathrm{C} & 2.54325 & -0.55471 & 4.05889 \\ \mathrm{H} & 3.38335 & -1.07106 & 3.55927 \\ \mathrm{H} & 1.77755 & -1.31806 & 4.26780 \\ \mathrm{C} & 3.00387 & 1.56757 & 2.80105 \\ \mathrm{H} & 3.85228 & 1.10648 & 2.26380 \\ \mathrm{H} & 2.56575 & 2.31429 & 2.12049 \\ \mathrm{C} & 3.49798 & 2.22806 & 4.08778 \\ \mathrm{H} & 2.65399 & 2.75083 & 4.57088 \\ \mathrm{H} & 4.25342 & 2.99543 & 3.85941 \\ \mathrm{C} & 3.03637 & 0.09766 & 5.34993 \\ \mathrm{H} & 3.46197 & -0.66114 & 6.02437 \\ \mathrm{H} & 2.17708 & 0.54366 & 5.88048 \\ \mathrm{C} & 4.06386 & 1.19172 & 5.05865 \\ \mathrm{H} & 4.96254 & 0.73192 & 4.61107 \\ \mathrm{H} & 4.38790 & 1.67469 & 5.99317 \\ & & & \end{array}$


Table S13

\section{M06-2X/def2-SVP}

$\mathrm{LiCl}$

ENERGY (WITHOUT THE ZERO-POINT) $=-467.628696004$ Hartree FREE ENERGY $=-467.647833$ Hartree

\begin{tabular}{lrrr} 
& Atomic & \multicolumn{3}{c}{ Coordinates (Angstroms) } \\
& Number & X & \multicolumn{2}{c}{ Y } \\
- & & & \\
$\mathrm{Li}$ & -0.50225 & 0.00000 & 0.00000 \\
$\mathrm{Cl}$ & 1.50225 & 0.00000 & 0.00000
\end{tabular}

\section{Table S14}

\section{M06-2X/def2-SVP}

$\mathrm{R}^{1} \mathrm{GeCl}\left(\mathrm{R}^{1}=\mathrm{PhC}(\mathrm{N} t \mathrm{Bu})_{2}\right)$

ENERGY (WITHOUT THE ZERO-POINT) $=-3231.00200237$ Hartree FREE ENERGY $=-3230.697892$ Hartree

\begin{tabular}{rrrr} 
& \multicolumn{2}{c}{ Atomic } & \multicolumn{3}{c}{ Coordinates (Angstroms) } \\
& Number & X & \multicolumn{2}{c}{ Y } & Z \\
\hdashline & & & \\
C & -0.14680 & 0.28871 & 1.55341 \\
C & -1.00234 & 1.41754 & 2.02950 \\
C & -2.61625 & 3.50569 & 2.93661
\end{tabular}




$\begin{array}{lrrr}\mathrm{C} & -2.08188 & 1.15100 & 2.87809 \\ \mathrm{C} & -0.73289 & 2.73050 & 1.63599 \\ \mathrm{C} & -1.54091 & 3.77277 & 2.09073 \\ \mathrm{C} & -2.88626 & 2.19383 & 3.32988 \\ \mathrm{H} & -2.28645 & 0.12223 & 3.18127 \\ \mathrm{H} & 0.11095 & 2.93228 & 0.97311 \\ \mathrm{H} & -1.32811 & 4.79703 & 1.78198 \\ \mathrm{H} & -3.72739 & 1.98312 & 3.99163 \\ \mathrm{H} & -3.24691 & 4.32204 & 3.29137 \\ \mathrm{~N} & -0.36681 & -0.42917 & 0.45998 \\ \mathrm{~N} & 0.92505 & -0.17578 & 2.18133 \\ \mathrm{Ge} & 1.22519 & -1.62592 & 0.80478 \\ \mathrm{C} & -1.28658 & -0.24992 & -0.66409 \\ \mathrm{C} & 1.69902 & 0.33685 & 3.31258 \\ \mathrm{C} & 2.39920 & 1.64276 & 2.91222 \\ \mathrm{H} & 2.94694 & 1.49410 & 1.97053 \\ \mathrm{H} & 1.66779 & 2.45130 & 2.77376 \\ \mathrm{H} & 3.10500 & 1.95493 & 3.69638 \\ \mathrm{C} & 0.84025 & 0.54744 & 4.56465 \\ \mathrm{H} & 1.48900 & 0.78760 & 5.41994 \\ \mathrm{H} & 0.27660 & -0.36689 & 4.80300 \\ \mathrm{H} & 0.13076 & 1.37612 & 4.43749 \\ \mathrm{C} & 2.76166 & -0.72921 & 3.59761 \\ \mathrm{H} & 3.39565 & -0.87923 & 2.70910 \\ \mathrm{H} & 2.28983 & -1.68770 & 3.86172 \\ \mathrm{H} & 3.40746 & -0.41611 & 4.42958\end{array}$




$\begin{array}{lccc}\mathrm{C} & -1.09481 & -1.48520 & -1.54967 \\ \mathrm{H} & -0.04853 & -1.54964 & -1.88892 \\ \mathrm{H} & -1.73926 & -1.42638 & -2.43763 \\ \mathrm{H} & -1.34439 & -2.40292 & -0.99593 \\ \mathrm{C} & -2.75052 & -0.16644 & -0.21743 \\ \mathrm{H} & -2.95567 & 0.75976 & 0.33603 \\ \mathrm{H} & -3.00708 & -1.02433 & 0.42189 \\ \mathrm{H} & -3.40652 & -0.18354 & -1.10028 \\ \mathrm{C} & -0.89325 & 1.00070 & -1.46236 \\ \mathrm{H} & 0.17790 & 0.96062 & -1.70731 \\ \mathrm{H} & -1.09104 & 1.91272 & -0.88190 \\ \mathrm{H} & -1.47562 & 1.05908 & -2.39400 \\ \mathrm{Cl} & 2.64326 & -0.35375 & -0.44490\end{array}$

Table S15

\section{M06-2X/def2-SVP}

TMS-CC-Li

ENERGY (WITHOUT THE ZERO-POINT) $=-492.588552063$ Hartree

FREE ENERGY = -492.503560 Hartree

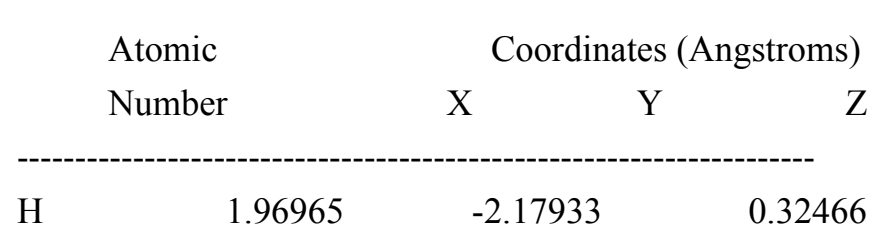




$\begin{array}{lccc}\mathrm{C} & 1.27266 & -1.90440 & 1.12991 \\ \mathrm{H} & 1.86601 & -1.68291 & 2.02917 \\ \mathrm{H} & 0.74207 & -0.98744 & 0.83062 \\ \mathrm{H} & -1.67894 & -1.89120 & 2.56985 \\ \mathrm{C} & -1.11521 & -2.79543 & 2.84636 \\ \mathrm{H} & -1.83442 & -3.59966 & 3.06030 \\ \mathrm{H} & -0.55744 & -2.58623 & 3.77098 \\ \mathrm{H} & -0.27022 & -3.95430 & -0.92139 \\ \mathrm{C} & -0.93492 & -3.65361 & -0.09824 \\ \mathrm{H} & -1.49561 & -2.76055 & -0.41442 \\ \mathrm{H} & -1.65201 & -4.46999 & 0.07234 \\ \mathrm{Si} & 0.06087 & -3.30936 & 1.46529 \\ \mathrm{C} & 0.97969 & -4.81692 & 1.96134 \\ \mathrm{C} & 1.59792 & -5.83221 & 2.29658 \\ \mathrm{Li} & 2.54581 & -7.39249 & 2.81368 \\ & & & \end{array}$

Table S16

\section{M06-2X/def2-SVP}

$\mathrm{R}^{2} \mathrm{GeCl}\left(\mathrm{R}^{2}=\mathrm{PhC}(\mathrm{NCy})_{2}\right)$

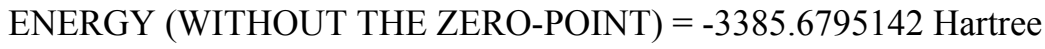

FREE ENERGY $=-3385.300473$ Hartree

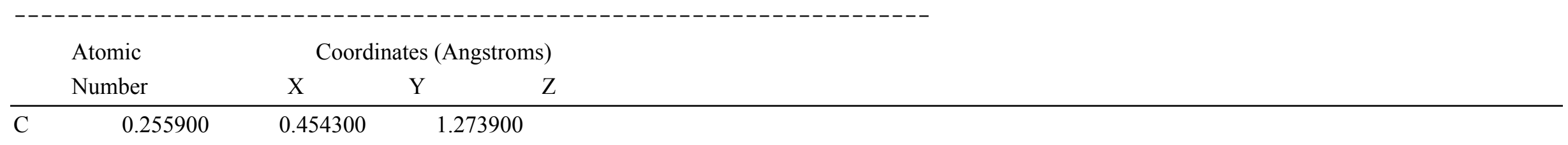




$\begin{array}{lrrr}\mathrm{C} & -0.809200 & 1.242100 & 1.953700 \\ \mathrm{C} & -2.791800 & 2.702500 & 3.263800 \\ \mathrm{C} & -1.965300 & 0.599900 & 2.409300 \\ \mathrm{C} & -0.645100 & 2.615100 & 2.161000 \\ \mathrm{C} & -1.639100 & 3.344000 & 2.810800 \\ \mathrm{C} & -2.952900 & 1.330900 & 3.066100 \\ \mathrm{H} & -2.078500 & -0.475200 & 2.253300 \\ \mathrm{H} & 0.262900 & 3.107600 & 1.806700 \\ \mathrm{H} & -1.512800 & 4.416300 & 2.965900 \\ \mathrm{H} & -3.851300 & 0.827900 & 3.426000 \\ \mathrm{H} & -3.567600 & 3.273700 & 3.775800 \\ \mathrm{~N} & 0.100400 & -0.229900 & 0.150000 \\ \mathrm{~N} & 1.495600 & 0.337800 & 1.725400 \\ \mathrm{Ge} & 2.056100 & -0.816000 & 0.156500 \\ \mathrm{C} & -1.071600 & -0.242300 & -0.706700 \\ \mathrm{H} & -1.888300 & 0.262000 & -0.166800 \\ \mathrm{C} & -1.499000 & -1.688200 & -0.997000 \\ \mathrm{H} & -2.501600 & -1.663600 & -1.457100 \\ \mathrm{H} & -1.580600 & -2.235500 & -0.045700 \\ \mathrm{C} & -0.832600 & 0.527600 & -2.015200 \\ \mathrm{H} & -0.470900 & 1.539900 & -1.776200 \\ \mathrm{H} & -1.807000 & 0.643700 & -2.519700 \\ \mathrm{C} & -0.532200 & -2.404700 & -1.940900 \\ \mathrm{H} & 0.437300 & -2.552600 & -1.434800 \\ \mathrm{H} & -0.907900 & -3.416200 & -2.155500 \\ \mathrm{C} & 0.132400 & -0.192700 & -2.956900\end{array}$




$\begin{array}{lrrr}\mathrm{H} & 1.140400 & -0.221200 & -2.508600 \\ \mathrm{H} & 0.230900 & 0.374100 & -3.894900 \\ \mathrm{C} & -0.335900 & -1.620700 & -3.238600 \\ \mathrm{H} & 0.381400 & -2.136700 & -3.894000 \\ \mathrm{H} & -1.294700 & -1.583500 & -3.785700 \\ \mathrm{C} & 2.024100 & 0.743300 & 3.008300 \\ \mathrm{H} & 1.228900 & 1.268400 & 3.568800 \\ \mathrm{C} & 2.451900 & -0.479400 & 3.827700 \\ \mathrm{H} & 3.199300 & -1.046300 & 3.244900 \\ \mathrm{H} & 1.591200 & -1.153200 & 3.951500 \\ \mathrm{C} & 3.198900 & 1.710200 & 2.829700 \\ \mathrm{H} & 3.966300 & 1.207500 & 2.214200 \\ \mathrm{H} & 2.864500 & 2.591700 & 2.260300 \\ \mathrm{C} & 3.799600 & 2.119800 & 4.173900 \\ \mathrm{H} & 3.048500 & 2.693600 & 4.744700 \\ \mathrm{H} & 4.655500 & 2.792600 & 4.016100 \\ \mathrm{C} & 3.045900 & -0.066700 & 5.173400 \\ \mathrm{H} & 3.362600 & -0.958500 & 5.734100 \\ \mathrm{H} & 2.265700 & 0.425800 & 5.780000 \\ \mathrm{C} & 4.218900 & 0.896300 & 4.989100 \\ \mathrm{H} & 5.034800 & 0.373200 & 4.461100 \\ \mathrm{H} & 4.620800 & 1.206100 & 5.965400 \\ \mathrm{Cl} & 1.622900 & -2.778900 & 1.204700 \\ -----------------1\end{array}$




\section{References}

[S1] G. M. Sheldrick, Acta Crystallogr., Sect. A: Found. Crystallogr., 1990, 46, 467.

[S2] G. M. Sheldrick, SHELXL-97, Program for Crystal Structure Refinement, University of Göttingen, Göttingen, Germany, 1997.

[S3] Y. Zhao and D. Truhlar, Theor. Chem. Acc. 2008, 120, 215.

[S4] F. Weigend and R. Ahlrichs, Phys. Chem. Chem. Phys. 2005, 7, 3297.

[S5] X. Li and M. J. J. Frisch, Chem. Theory Comput. 2006, 2, 835.

[S6] M. J. Frisch, G. W. Trucks, H. B. Schlegel, G. E. Scuseria, M. A. Robb, J. R. Cheeseman, G. Scalmani, V. Barone, G. A. Petersson, H. Nakatsuji, X. Li, M. Caricato, A. V. Marenich, J. Bloino, B. G. Janesko, R. Gomperts, B. Mennucci, H. P. Hratchian, J. V. Ortiz, A. F. Izmaylov, J. L. Sonnenberg, D. Williams-Young, F. Ding, F. Lipparini, F. Egidi, J. Goings, B. Peng, A. Petrone, T. Henderson, D. Ranasinghe, V. G. Zakrzewski, J. Gao, N. Rega, G. Zheng, W. Liang, M. Hada, M. Ehara, K. Toyota, R. Fukuda, J. Hasegawa, M. Ishida, T. Nakajima, Y. Honda, O. Kitao, H. Nakai, T. Vreven, K. Throssell, J. A. Montgomery, Jr., J. E. Peralta, F. Ogliaro, M. J. Bearpark, J. J. Heyd, E. N. Brothers, K. N. Kudin, V. N. Staroverov, T. A. Keith, R. Kobayashi, J. Normand, K. Raghavachari, A. P. Rendell, J. C. Burant, S. S. Iyengar, J. Tomasi, M. Cossi, J. M. Millam, M. Klene, C. Adamo, R. Cammi, J. W. Ochterski, R. L. Martin, K. Morokuma, O. Farkas, J. B. Foresman and D. J. Fox, Gaussian, Inc., Wallingford CT, 2016.

[S7] A. V. Marenich, C. J. Cramer, D. G. Truhlar, J. Phys. Chem. B, 2009, 113, 6378. 